\title{
Development of Very-High-Frequency Field-Intensity Standards
}

\author{
By Frank M. Greene and Max Solow
}

\begin{abstract}
A description is given of the development of two very-high-frequency field-intensity standards, which are being used at this Bureau for the calibration of commercial fieldintensity sets in the range 30 to 300 megacycles. These standards are employed to establish known values of field intensity by either of two methods: (a) the standard-antenna method in which the open-circuit voltage at the center of a receiving dipole is measured directly; (b) the standard-field method in which the current at the center of a transmitting dipole is accurately known. The techniques used for determining the antenna current and voltage are described.

The current distribution on the antenna is determined theoretically, using Schelkunoff's method, which gives the effective length. These values are compared with those obtained by measurement.

Results of field tests at 100 megacycles are presented in which the above two methods were directly intercompared using horizontal polarization. Their accuracy and limitations are discussed.
\end{abstract}

\section{Introduction}

For a number of years the National Bureau of Standards has maintained among its other public services, the calibration of commercial field-intensity meters. These instruments are largely used by engineering consultants and broadcast engineers, in order to determine a radio station's antenna efficiency and coverage area, acceptable values of which are specified by the Federal Communications Commission. The frequency range of this calibration service has in the past extended to approximately $30 \mathrm{Mc}$ and is maintained by the Bureau's Central Radio Propagation Laboratory.

More recently, as a result of the greatly increased use of the very-high-frequency (VHF) band (30 to $300 \mathrm{Mc}$ ) for frequency-modulation and television broadcasting and other communication purposes, the Central Radio Propagation Laboratory has undertaken to extend the frequency range of this calibration service to include these frequencies.
This necessitated the development of accurate field-intensity standards for calibration purposes. Such standards are usually employed to determine a value of field intensity in terms of either a known voltage or current in an antenna, together with certain geometrical relationships involved, such as the antenna length, distance of separation, etc. The standards developed are similar in principle to those already in use at the lower frequencies. However, it was necessary to modify the techniques employed for the measurement of antenna current and voltage as well as the over-all technique in the use of the standards in order to adapt them to use at the higher frequencies.

In many instances the accuracy with which the value of a physical quantity may be determined increases with the simplicity of the method used to make the determination. Such measurements usually involve a theoretical calculation, or an experimental observation, or both. In establishing the field-intensity standards discussed herein, the methods adopted for current and voltage measure-

${ }^{*} \mathrm{~A}$ summary of this paper was presented at the joint meeting of the International Scientific Radio Union and the Institute of Radio Engineers at Washington, D. C., on May 2, 1949. 
ment were purposely made as elementary as possible for this reason. It is believed that this enables the achievement of the maximum accuracy possible or practicable at the present time. This of necessity results in some limitation of the range of measurement and flexibility of use, but the premium paid results in increased accuracy.

The standards or methods used to establish accurately known values of VHF field-intensity are (a) the standard-antenna method and (b) the standard-field method.

It is the purpose of this paper to describe two such experimental standards in some detail, the techniques involved for measuring the antenna voltage or current, as well as the propagation tests made to intercompare the field-intensity values obtained by both methods. The accuracy and limitations of the two methods are also discussed.

This work done by the authors represents one phase of a program for the development of accurate VHF field-intensity calibration standards. Although these standards were developed to cover the entire VHF band, the initial propagation tests described herein were made at a frequency of $100 \mathrm{Mc}$, using horizontal polarization only; and the standard antennas were horizontal halfwave self-resonant dipoles in all cases.

No claims are made as to the originality of the individual methods or techniques used, as most of these have been reported elsewhere. However, the standards discussed here are novel at least in their simplicity and in the accuracy obtainable. Practical rationalized mks units are used throughout this paper.

\section{Standard-Antenna Method}

\section{Relation Between Field-Intensity and Induced Voltage}

The magnitude of the electric component of VHF field-intensity existing at a given point in space may be determined in terms of the voltage, $V_{o c}$, induced in a standard receiving dipole immersed in the field, together with the antenna geometry. It will be assumed in the following that the antenna is a horizontal half-wave dipole oriented for maximum response, and that the voltage is referred to the center terminals. In case the antenna is not open-circuited, the voltage measured, $V_{L}$, is of course not the induced voltage but is related to it by the voltage-transfer ratio,

$$
\eta \equiv\left|\frac{V_{L}}{V_{o c}}\right|=\left|\frac{Z_{L}}{Z_{A}+Z_{L}}\right|,
$$

where $Z_{L}$ is the load impedance, in ohms, connected to the antenna terminals, and $Z_{A}$ the antenna input impedance in ohms.

The magnitude of the electric component of field-intensity (in volts per meter) is related to the induced voltage by

$$
|E|=\frac{V_{o c}}{l_{H}},
$$

where $l_{B}$ is the effective length of the antenna in meters. ${ }^{1}$

\section{Effective Length of the Antenna}

Assuming a sinusoidal current distribution on the antenna, the effective length (in meters) is

$$
l_{H}=\frac{\lambda}{\pi} \tan \frac{\pi l}{\lambda},
$$

where $\lambda$ is the wavelength in meters, and $l$ the antenna half-length in meters.

For a half-wave dipole the effective length (in meters) reduces to

$$
l_{H}=\frac{\lambda}{\pi}
$$

As is known, the current distribution on an antenna is not exactly sinusoidal except for an infinitely thin filament. For cylindrical antennas of radius $a$ and half-length $l$, the departure from a sinusoidal distribution becomes progressively greater as the ratio $2 l / a$ decreases (i. e., as the antenna gets fatter).

An approximate solution for the free-space current distribution on a cylindrical transmitting antenna ${ }^{2}$ has been obtained by Schelkunoff [1] ${ }^{3}$ among others, and is given by the sum of eq 34 and 35 in the appendix. The relative distribution

\footnotetext{
1 It is assumed here (and our results seem to indicate) that the relative current distribution and hence the effective length is not (to a first approximation) a function of either the height of the antenna above the ground or the terminating impedance, $Z_{L \text {. }}$.

${ }^{2}$ How this is handled in the case of the receiving antenna has not been made too clear in the literature, but the difference between the two cases is apparently small for moderately thin antennas. The reader is referred, for instance, to J. C. Slater, Microwave transmission, pp. 219 to 230 (McGraw-Hill Book Co., Inc., New York, N. Y., 1942).

${ }^{3}$ Figures in brackets indicate the literature references at the end of this paper.
} 


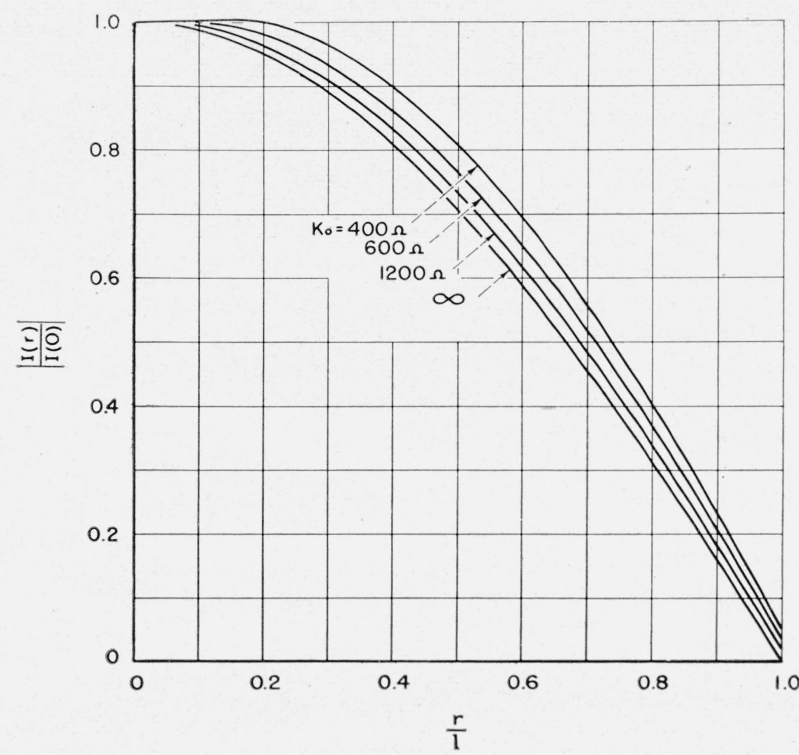

FIGURE 1. Theoretical free-space relative current distributions on a cylindrical half-wave dipole as determined from Schelkunoff's analysis.

The relative current $I(r) / I(0)$ is shown versus the fractional half-length $r / l$ for several values of the average characteristic impedance, $K_{0}=120\left[\log _{\bullet}(2 l / a)-1\right]$, as determined from the sum of eq 34 and 35 in the appendix.

is a function of both the phase length $2 \beta l$, and the ratio $2 l / a$, and may be represented by

$$
\frac{I(r)}{I(0)}=f\left(\beta l, K_{0}\right)
$$

where

$I(r)=$ current in amperes at a distance $r$ meters from the center of the antenna $I(0)=$ current in amperes at the center of the antenna $(r=0)$

$K_{0} \equiv 120\left(\log _{e} \frac{2 l}{a}-1\right)$ ohms, and is the average characteristic impedance over the half-length $l$

$$
\beta=2 \pi / \lambda \text {. }
$$

The relative current distributions as determined from the magnitude of the sum of eq 34 and 35 are shown in figure 1 for half-wave dipoles for several values of $K_{0}$. Because of the increasing complexity of the higher order Bessel functions in the infinite series of eq 35 , only the first two terms of this series were evaluated. This accounts for the fact that the current as shown in figure 1 does not reduce quite to zero as it should at the end of the antenna, $r / l=1.0$. The contribution from the neglected terms in this series is appreciable only near the ends of the antenna, so that this approximation does not appreciably affect our results here.

The effective length of the antenna (in meters) may be determined from the relative current distribution by the relation

$$
l_{H}=\int_{-l}^{l}\left|\frac{I(r)}{I(0)}\right| d r
$$

The magnitude of the sum of eq 34 and 35 may be substituted in eq 6 and the integration performed either mathematically or mechanically. We preferred the latter as being less involved.

In this manner a number of values of effective length, $l_{H}$, were determined for various values of the average characteristic impedance, $K_{0}$, between 400 and 1,200 ohms. The percentage increase in $l_{H}$ over that of an infinitely thin filament, $K_{0}=\infty$, is shown versus $K_{0}$ in figure 2 for half-wave dipoles. ${ }^{4}$

The agreement between calculated and observed values of $l_{H}$ for the antenna used will be discussed in section IV.

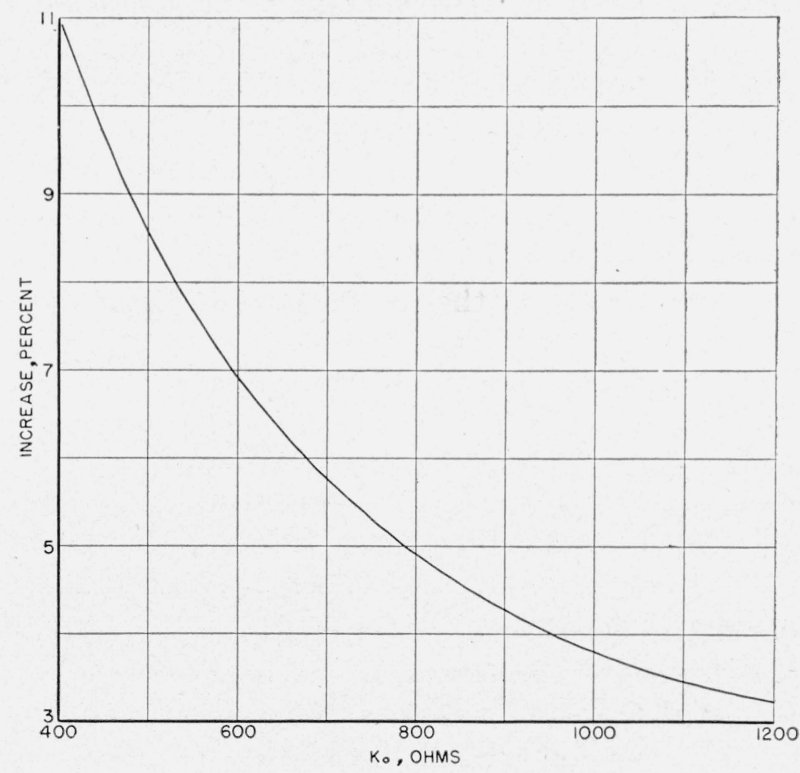

Figure 2. Percentage increase in effective length of a cylindrical half-wave dipole (over that of an infinitely thin filament) versus the average characteristic impedance, $K_{0}$, as determined by substituting the sum of eq 34 and 35 in 6 .

4 The percentage increase in $l_{H}$ for the case of thin self-resonant dipple antennas was found to be very nearly the same as that for full half-wave dipoles for the values of $K_{0}$ usually encountered. 
In order to operate a half-wave dipole at selfresonance it is necessary, as is known, to shorten its length somewhat from a full half-wavelength.

Schelkunoff [2] and King [3] have obtained independent approximate solutions yielding the required shortening for self-resonant operation of thin cylindrical antennas. Although obtained by radically different methods, the value of the required shortening as determined from Schelkunoff's solution is in substantial agreement with that obtained from King's second-order solution, and is given by

$$
\frac{4 l}{\lambda}=1-\frac{60 S i(2 \pi)}{\pi K_{0}}=1-\frac{27.08}{K_{0}}
$$

where $\operatorname{Si}(x) \equiv \int_{0}^{x}(\sin t / t) d t$ and $\operatorname{Si}(2 \pi)=1.41815$.

Values of the percentage shortening determined from eq 7 are shown versus the average characteristic impedance of the antenna $K_{0}$, in figure 3 .

\section{Antenna Voltmeter}

The standard receiving antenna used was a selfresonant (half-wave) dipole shortened approximately 4 percent from a full half-wavelength $(f=$ $100.0 \mathrm{Mc}$ ) (as determined from fig. 3) to make it self-resonant. Dural tubing $3 / 16$ in. in diameter was used, giving a ratio $2 l / a \cong 600$, and an average characteristic impedance $K_{0}=120 \quad\left[\log _{e}(2 l / a)-1\right]$ $\cong 650 \mathrm{ohms}$. The induced voltage referred to the center terminals of the antenna was measured directly by means of a relatively high-impedance balanced voltmeter connected across the gap at the center. In this manner the necessity for a separate measurement of the antenna input impedance was eliminated, which greatly simplified the problem. The voltmeter consisted of a modified type $1 \mathrm{~N} 28$ silicon-crystal rectifier built into the gap as shown in figure 4 . The crystal output was filtered by means of a balanced resistancecapacitance network, and the d-c output voltage was measured directly against a standard cell on a special precision slide-wire potentiometer. The central portion of an experimental receiving antenna assembly minus the antenna rods is shown in figure 5. The input impedance of the resistance-capacitance filter was approximately 10,000 ohms in shunt with $0.05 \mu \mu \mathrm{f}$. Highfrequency resistors were employed, which were available commercially.

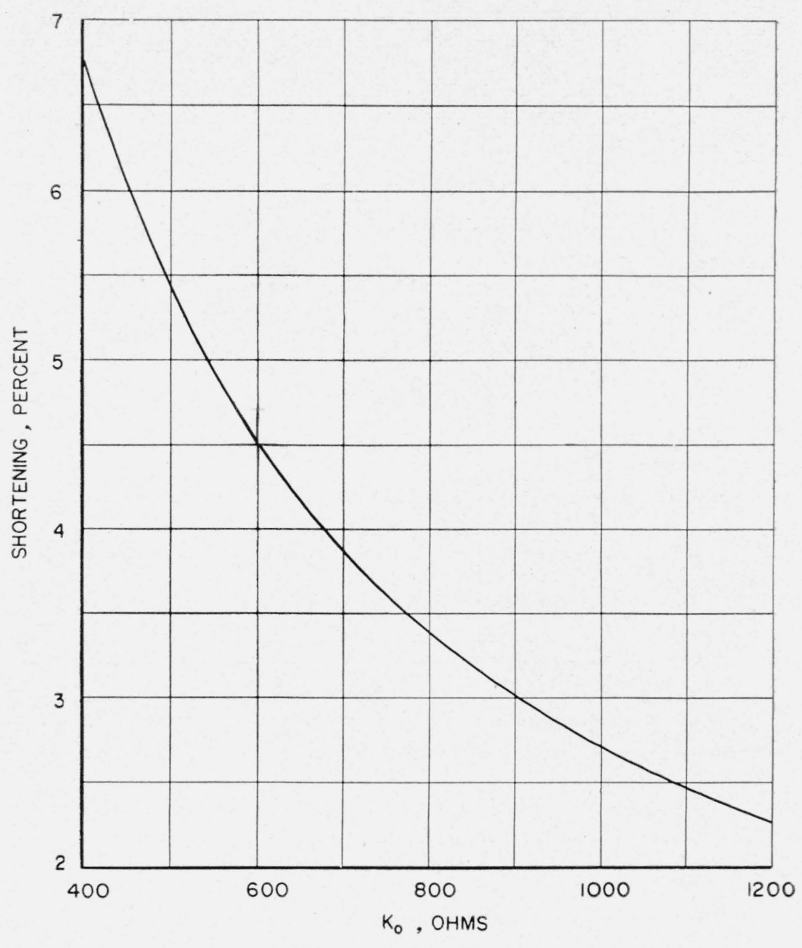

Figure 3. Percentage shortening of a cylindrical half-wave dipole required for self-resonant operation versus the average characteristic impedance, $K_{0}$, as determined from $e q \%$.

The effective shunt capacity of the crystal diode was less than $0.75 \mu \mu \mathrm{f}$. However, since the resulting reactance of some 2,000 ohms was in quadrature with the radiation resistance of the antenna, the resulting shunting error from this source was a small fraction of 1 percent.

In selecting the crystal rectifier it was necessary to consider both its $\mathrm{r}-\mathrm{f}$ (input) resistance and its
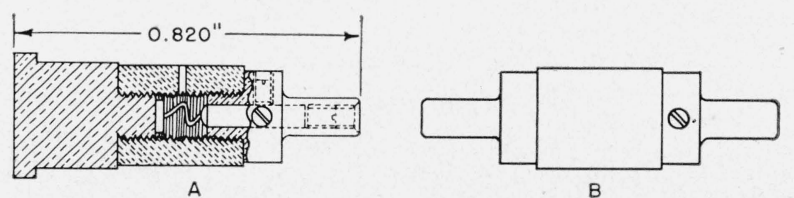

B

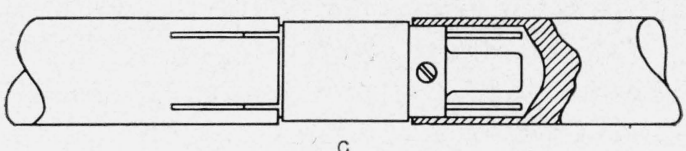

FIgure 4. Method used to mount the type 1 N28 siticon crystal rectifier in the gap at the center of the half-wave standard receiving antenna.

A, Western Electric $1 \mathrm{~N} 28$ silicon crystal rectifier; B, 1 N28 as modified; C, modified $1 \mathrm{~N} 28$ as mounted in the antenna gap. 


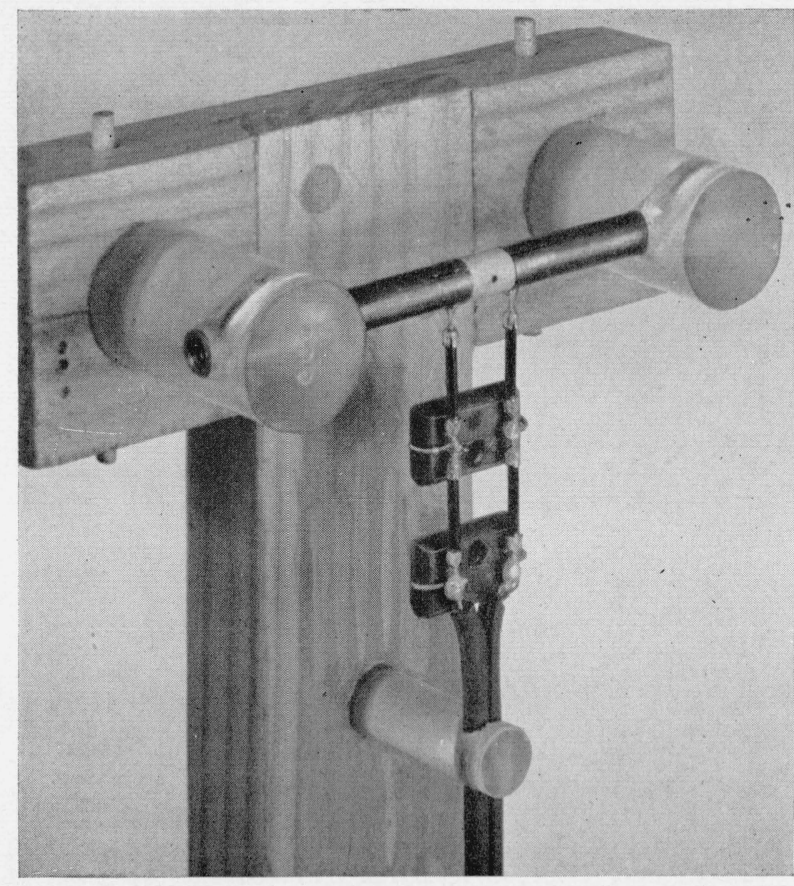

Figure 5. View of the central portion of an experimental standard receiving antenna minus the antenna rods showing the crystal rectifier and resistance-capacity filter network.

(internal) d-c-output resistance. Both are a function of the applied r-f-voltage level, decreasing with increasing level, although not necessarily at the same rate.

In order to limit the shunting caused by the presence of the crystal voltmeter in determining the open-circuit antenna voltage, it was necessary to select a crystal having as high a value of $\mathrm{r}-\mathrm{f}$ resistance as possible. On the other hand, to maintain as high a sensitivity as possible in measuring the d-c-output voltage of the crystal, it was necessary to select a crystal with a relatively low value of d-c-output resistance.

A satisfactory compromise was obtained by selecting a crystal having an $\mathrm{r}-\mathrm{f}$ resistance between 8,000 and 10,000 ohms when measured at an r-f level of $0.1 \mathrm{v}$ and self-biased. This resulted in a shunting error of less than 2 percent in determining the induced antenna voltage. It was found in general that the type $1 \mathrm{~N} 28$ crystals came closest to meeting these requirements.

The variation of both the $\mathrm{r}-\mathrm{f}$ resistance and the d-c-output resistance versus applied r-f voltage at $100 \mathrm{Mc}$ is shown in figure 6 for the 1 N28 crystal selected when operated self-biased. These crystal characteristics are not representative of the 1 N28 crystals in general, as it was possible to find many with considerably higher or lower values of $r-f$ and d-c resistance than the values shown. The variation among other crystals of types $1 \mathrm{~N} 21$ to $1 \mathrm{~N} 27$ was found to be even greater.

The $\mathrm{r}-\mathrm{f}$ input resistance of the $1 \mathrm{~N} 28$ crystal was measured by a resistance-variation method [4] using several high-frequency resistors having widely separated values as cross checks in determining each value of crystal resistance. The crystal was connected in series with a $200-\mu \mu \mathrm{f} \mathrm{d-c-}$ blocking condenser across an antiresonant circuit during these measurements. The d-c-output voltage acting as self-bias on the crystal was developed across this condenser.

The d-c-output resistance of the crystal is the equivalent internal dynamic source resistance of the d-c-output circuit. This is sometimes referred to as the d-c, or video, impedance [5] and may be defined (in ohms) as

$$
R_{i} \equiv \frac{\Delta e}{\Delta i}
$$

where $\Delta e=\mathrm{a}$ small change in the d-c balancing voltage of the slidewire potentiometer and $\Delta i=$ the resulting change in the $d$-c current in the output circuit. If $\Delta e$ is kept smaller than about $5 \mathrm{mv}$, when measuring $R_{i}$, either a positive or a negative value of $\Delta e$ will result in very nearly the same value of $R_{i}$.

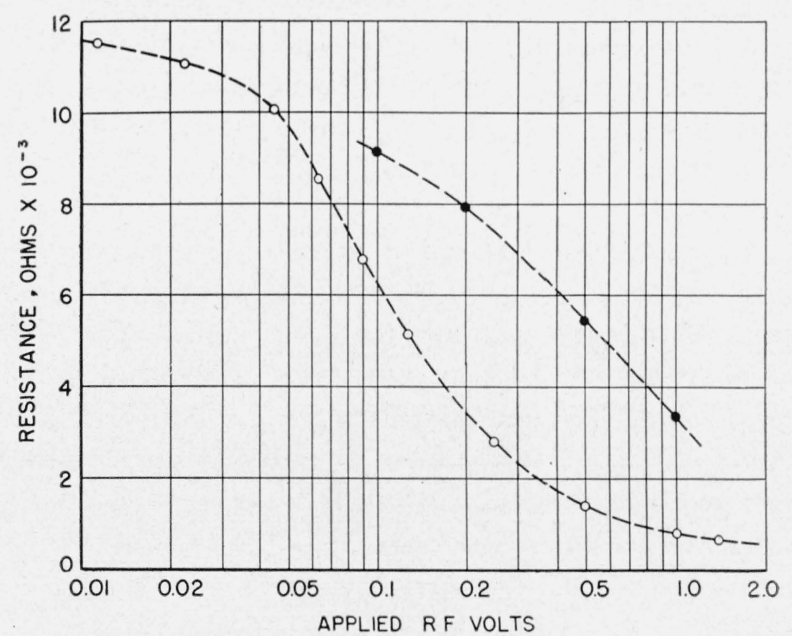

FIGURE 6. Variation of the $r$-f input resistance and dynamic $d-c$ output resistance as a function of the applied $r-f$ voltage level for a selected type 1 N28 silicon crystal rectifier.

The measurements were made at $100 \mathrm{Mc}$ with full self $\mathrm{d}-\mathrm{c}$ bias applied to the crystal. These curves are not representative of the type 1 N28 crystals in general. $\bigcirc$, d-c output resistance;, $\mathrm{r}$-f resistance. 
The d-c output resistance of the crystal was determined in this manner for a number of $\mathrm{r}-\mathrm{f}$ voltage levels. The crystal was removed from the antenna and placed in a coaxial mount fed by a 25-ohm r-f source. The d-c output voltage was measured across a $200-\mu \mu \mathrm{f}$ blocking condenser in the ground-return circuit.

As the d-c output voltage of the crystal was measured on a precision slidewire potentiometer, it is necessary to define the sensitivity of this instrument. This determined the useful range of d-c output voltages that could be measured, and hence the resulting range of applied $r-f$ voltages that could be determined.

The voltage sensitivity of the potentiometer (in volts) is

$$
\Delta e=R \Delta i
$$

where

$\Delta e=$ smallest change in the balancing voltage that can be set on the slidewire dial of the potentiometer.

$\Delta i=$ smallest unbalance current readable on the galvanometer.

$R=$ total series $\mathrm{d}$-c resistance of the measuring circuit and crystal.

The potentiometer used in these experiments was a commercially available portable type, weighing approximately $15 \mathrm{lb}$. It housed a standard cell, dry cells for comparison purposes, and a sensitive light-beam galvanometer, together with the slidewire dial and standard resisters all in a portable carrying case. The instrument had a useful measuring range of 0.5 to $161 \mathrm{mv}$. Other instruments were available having a range up to $1.6 \mathrm{v}$.

The most sensitive portable light-beam galvanometer available was used for measuring the output voltage of the crystal. Its sensitivity was approximately $0.03 \mu$ a per scale division $(1 \mathrm{~mm})$. Assuming that one could detect a change of current, $\Delta i$, of 0.1 division, and that the total series d-c resistance of the crystal, filter, and potentiometer was approximately $20,000 \mathrm{ohms}$, the voltage sensitivity was $\Delta e=R \Delta i=2 \times 10^{4} \times 3 \times 10^{-9}=$ $60 \times 10^{-6}$ v. Thus, neglecting the other errors in the instrument, which were small, d-c output voltages of the crystal could be determined to an accuracy of better than 2 percent from something less than $3 \mathrm{mv}$ to over $1 \mathrm{v}$ (the safe upper voltage limit of the crystal). This corresponded to a range of $r-f$ input voltage to the crystal of roughly 0.02 to $1.0 \mathrm{v}$ as will be shown.

The silicon crystal voltmeter was calibrated in terms of a standard voltmeter. One type used at $100 \mathrm{Mc}$ comprised a quarter-wavelength balanced transmission line of accurately known characteristic impedance terminated in a previously calibrated VHF vacuum thermocouple. ${ }^{5}$ As is known, the magnitude of the transfer impedance of a quarter-wave length uniform lowloss transmission line is equal to its characteristic impedance [6], i. e.,

$$
\left|Z_{T}\right| \cong\left|\frac{V_{s}}{I_{R}}\right| \cong Z_{0}
$$

where

$$
\begin{aligned}
& V_{S}=\text { sending-end voltage } \\
& I_{R}=\text { receiving-end current } \\
& Z_{0}=\text { characteristic impedance. }
\end{aligned}
$$

Thus the sending-end voltage may be determined in terms of the receiving-end current and the characteristic impedance of the transmission line. This relationship is independent of the magnitude or phase-angle of the terminating impedance (in this case the heater of the VHF vacuum thermocouple). The line losses involved were found to be small enough so as not to appreciably affect the results.

The frequency error of the thermocouple was determined in terms of small bead-type thermistors used as current standards. These are known to maintain their d-c value of resistance up to frequencies well above the VHF band when biased to low values of resistance. The circuit used to determine the frequency error is shown in figure 7. The bridge circuit measured the d-c resistance of the series combination of the thermistors and thermocouple heater biased with either d-c or $r-f$ current. A measure of the $r-f$ current was thus provided in terms of a known d-c current. A negligibly small d-c measuring current was used in the presence of the r-f current so as not to appreciably alter the thermistor resistance. Two matched thermistors were used to preserve the symmetry, as the frequency correction of the thermocouple will in general be considerably different if used in an unbalanced circuit.

The desired value of direct current was first established in the thermocouple heater. Resist-

${ }_{5}^{5}$ The VHF thermocouple referred to herein has a separate heater insulated from the couple by means of a small glass bead. 


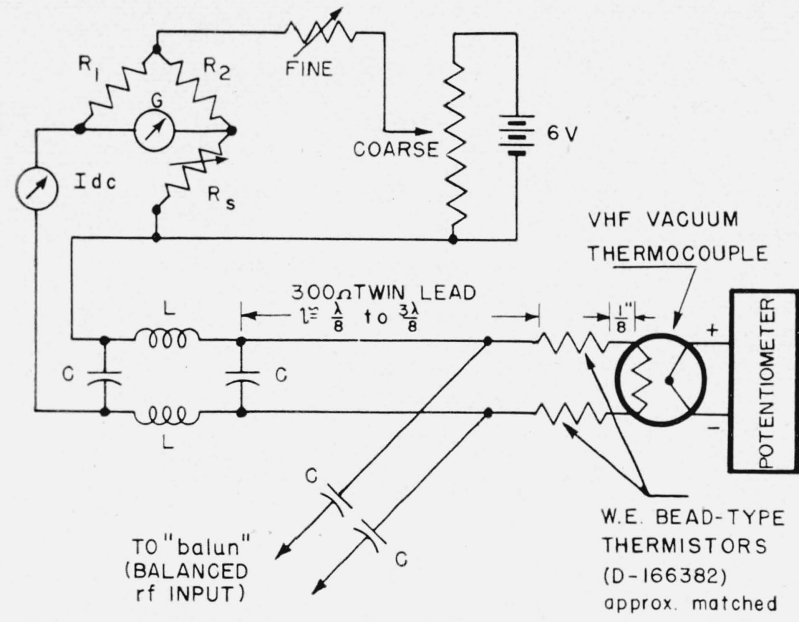

Figure 7. Circuit diagram of the apparatus used to determine the frequency correction of VHF vacuum thermoelements when used in a balanced circuit arrangement.

$\mathrm{L} \simeq 1 / 4 \mu \mathrm{h} ; \mathrm{C} \simeq 200 \mu \mu \mathrm{f}(\mathrm{f}=100 \mathrm{Mc})$.

ance $R_{s}$ was then adjusted to balance the bridge, at the same time maintaining the value of current by readjusting the battery voltage if necessary. After measuring the $d-c$ voltage output of the themocouple, the direct current was reduced in value to 5 percent or less. The $\mathrm{r}-\mathrm{f}$ voltage of desired frequency was then applied, adjusted in value until the bridge was rebalanced, and the d-c voltage output of the thermocouple measured. The r-f voltage was then removed and the value of direct current determined to reproduce this value of thermocouple output. The frequency correction was obtained from the two values of direct current. With sufficient sensitivity in the bridge and associated controls, it was found possible to reproduce these frequency corrections to a small fraction of a percent.

Generally speaking, for a given accuracy, a current standard is somewhat easier to provide at these frequencies than is a voltage standard. Fortunately, the relation given by eq 10 enables one to provide an $\mathrm{r}-\mathrm{f}$ voltage standard having an accuracy approximating that of the current standard used. The difficulty, however, in this case is in the restricted frequency range over which the standard may be used. Normally a continuous voltage calibration is not required over the frequency range of the standard antenna. A number of individual calibrations spaced at frequency intervals of approximately 25 or 50 percent over the desired range is usually sufficient when the response is broadband.
Probably one of the most accurate methods of determining the characteristic impedance of the balanced low-loss transmission line used in this voltage standard is from the relation

$$
Z_{0}=\frac{1}{V_{p} C}
$$

where

$$
\begin{aligned}
& Z_{0}=\text { characteristic impedance in ohms, } \\
& V_{p}=\text { velocity of propagation in meters per } \\
& \quad \text { second, and } \\
& C=\text { mutual capacity between conductors in } \\
& \quad \text { farads per meter. }
\end{aligned}
$$

The type of transmission line used was commercially available unshielded twin-lead having a nominal characteristic impedance of $75 \mathrm{ohms}$. The velocity of propagetion was determined to an accuracy of better than 0.25 percent at $100 \mathrm{Mc}$ by measuring the resonant frequency of a halfwavelength section of line short circuited at both ends. The length of the short circuit used was such as to introduce a negligible error in the measurement. The calculated effect of the line loss on the velocity of propagation was also found to be negligible. The balanced mutual capacity, $C$, per unit length was determined on a special precision Schering bridge at a frequency of 1,000 cycles to the same order of accuracy. There is no reason to suspect that the capacity per unit length should change with frequency, at least up to frequencies of several hundred megacycles. Thus the characteristic impedance was probably determined to better than 0.5 percent. The characteristic impedance of a number of samples $1 \mathrm{~m}$ in length cut from the same piece of twin-lead varied less than \pm 0.1 percent from the average value of the lot. This would seem to indicate the exceedingly small departure from uniformity existing within a given sample of the line. The average value of $Z_{0}$ for the lengths measured was $83.0 \mathrm{ohms}$. The variations between samples of different production-runs of twin-lead was quite large, being as high as 10 to 15 percent, as might be expected. The aging of the twin-lead seems to be negligible if not exposed to the weather or undue stress. This was indicated by having a value of the velocity of propagation of a particular sample repeat to within 0.1 percent after several months. 
Another type of balanced voltage standard used consisted simply of a sensitive thermocouple previously calibrated in a manner similar to that already described and shown in figure 7. However, in this case a single thermistor was used in shunt with the thermocouple heater. A 1,000$\mu \mu \mathrm{f}$ ceramic disk condenser was used in series with each thermocouple heater lead to provide dc isolation and preserve the symmetry. The leads were kept as short as physically possible ( $1 / 8$ in. in most cases).

The voltage standards described above were used at only one voltage level (approximately $1 \mathrm{v})$, that corresponding to the rated current through the terminating thermocouple. The voltage range was extended downward by means of a precision piston (mutual inductance) attenuator [6a] having an accuracy better than $0.1 \mathrm{db}$ (approximately $1.0 \%$ ) over a range of $60 \mathrm{db}$. The total rms harmonic content of the r-f generator output was less than 1 percent, so that any resulting error in the voltage standard from this cause was negligible. Pi matching networks were used in both the input and output circuits of the standard attenuator to decrease the insertion loss.

Figure 8 is a block diagram showing the equipment and its arrangement for one of the methods used to calibrate the balanced crystal voltmeter. The antenna rods were removed during calibration. A broadband balun [7] was used to transform from the unbalanced output of the standard attenuator to the balanced circuit of

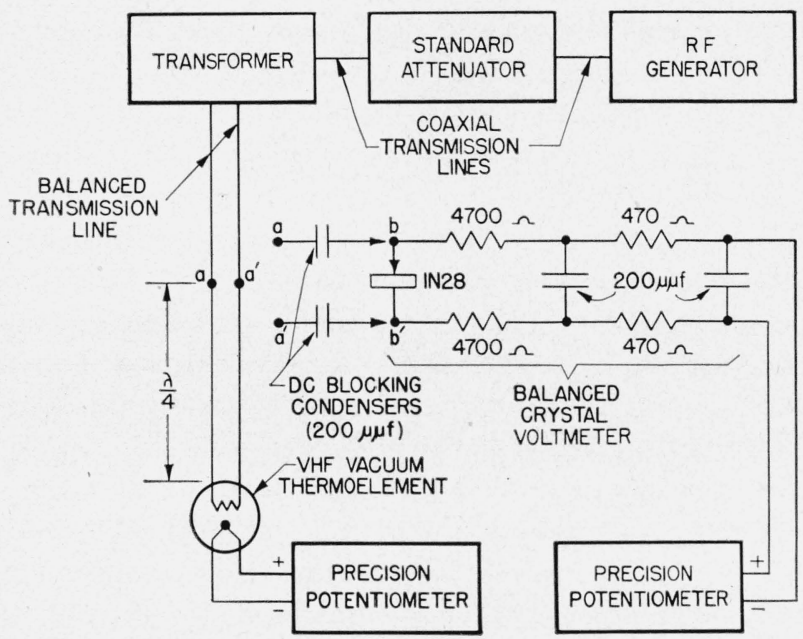

Figure 8. Diagram showing the equipment and method used to calibrate the balanced crystal voltmeter used in the standard receiving antenna.

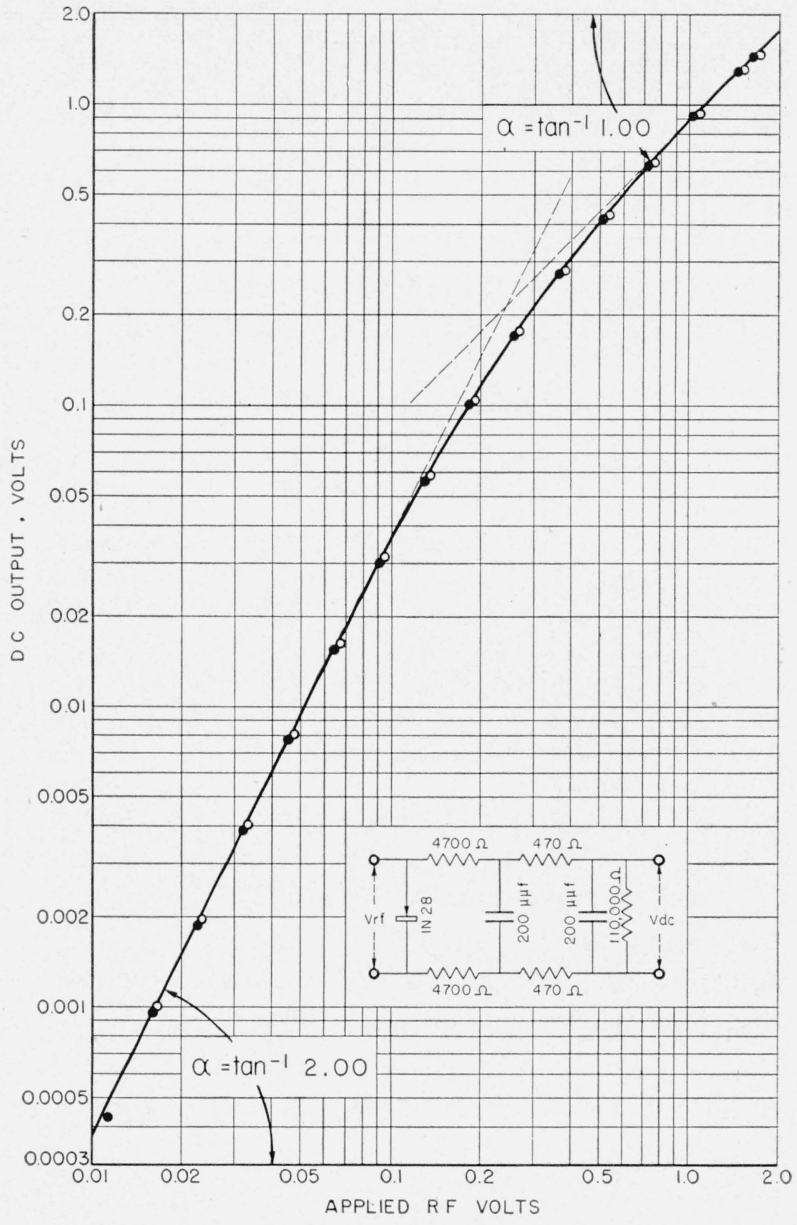

Figure 9. Typical calibration curves of the crystal voltmeter, showing the variation in $d$-c output voltage versus applied $r$-f voltage for the circuit shown.

Calibrations "A" and "B" were made 2 days apart at approximately the same ambient temperature. Calibration $\mathrm{A}$; $\bigcirc$, calibration $\mathrm{B}, f-100 \mathrm{Mc}$.

the antenna and voltmeter. The "Pi" matching networks are not shown. Two 200- $\mu \mu \mathrm{f}$ d-c blocking condensers were used to prevent shortcircuiting the d-c output of the antenna crystal.

Typical calibration curves of the antenna voltmeter made at $100 \mathrm{Mc}$ are shown in figure 9 . The usable range of $\mathrm{r}-\mathrm{f}$ input voltage was from 0.02 to $1.0 \mathrm{v}$ and the probably accuracy about \pm 2 percent over most of the range. The solid points and curve joining them represent the first of two calibrations. The circles represent a repeat calibration 2 days later at a temperature $1 \mathrm{deg} \mathrm{C}$ lower.

The data of figure 9 show the order of reproducibility possible with such a crystal voltmeter under partially controlled temperature conditions. The 
diameter of the individual points represents approximately 5 percent of the voltage, so that any point touching the curve is in agreement to within $\pm 2 \frac{1}{2}$ percent.

It was usually possible to repeat a crystal calibration to within 1 percent if made within a few hours at the same ambient temperature. In no case was the calibration relied on for more than a day.

In making the field-intensity measurements described in section IV, using this standard-antenna, the crystal voltmeter was always calibrated in the field within an hour of the time the tests were completed. A record of the temperature was kept and usually did not vary more than $2 \mathrm{deg} \mathrm{C}$ during any test. Thus all that was required of the crystal voltmeter was a good short-time stability.

Although the effect of variations in temperature and the accompanying hysteresis are known to contribute largely to the instability in a crystal voltmeter, no data were accumulated on the exact magnitude of these effects. We have so far avoided this by operating under as smal] a temperature variation as possible. Thus it would probably not be feasible to calibrate the crystal in a laboratory and use it for measurements outside at a temperature as much as 10 or $15 \mathrm{deg} \mathrm{C}$ different.

The response of the antenna crystal voltmeter was found to be essentially independent of frequency up to $200 \mathrm{Mc}$ with a rising response beyond (output indication high) due to series resonance within the crystal and mount. The series-resonant frequency for this voltmeter was of the order of $2,000 \mathrm{Mc}$.

\section{Standard-Field Method}

\section{Theory}

A predetermined value of field intensity may be established at a given point in space in terms of the current distribution in a transmitting antenna, the effect of the ground, and the geometry involved.

In the development of the standards being reported here, only horizontally polarized transmission over plane homogeneous earth having finite values of relative dielectric constant, $\epsilon_{r}$, and conductivity, $\sigma$, was considered. The distance of separation used between the transmitting and receiving antennas was from 2 to 20 wavelengths. These conditions simplify the resulting formulas somewhat and for the accuracy desired are quite justified. This range of distances is usually of little interest to the propagationist, at these frequencies, and many of the transmission vagaries of concern to him are ignored here. At these smaller separations a more exact knowledge of the actual angle of incidence and ground reflection coefficient is usually needed in order to produce the required degree of accuracy in predicting the actual value of field intensity existing. The geometry of the transmission system is shown in figure 10 .

The root-mean-square value of the electric component of field intensity produced by a horizontal transmitting dipole at distances greater than about $2 \lambda$ over plane homogeneous earth is $[8,9,10]$ (in volts per meter)

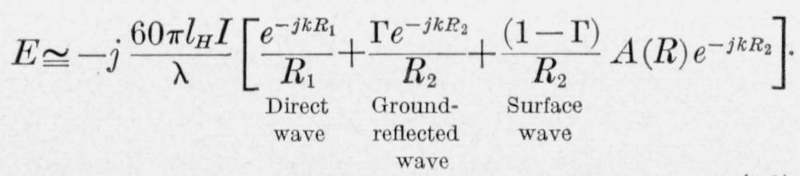

in which

$l_{H}=$ effective length of the antenna in meters

$\lambda=$ wavelength in meters

$R_{1}=$ direct-ray path length in meters

$R_{2}=$ ground-reflected-ray path-length in meters

$\Gamma=\rho e^{-j_{\phi}}=$ complex plane-wave reflection coefficient (horizontal polarization)

$A(R)=$ complex surface-wave attenuation factor

$I=\mathrm{rms}$ current in amperes at the center of the transmitting antenna

$k=2 \pi / \lambda$
$j=\sqrt{-1}$

The first term of eq 12 represents the field intensity that would exist if the transmitting and receiving antennas were located in free space. The remaining terms take into account the presence of the earth. The second term represents the ground-reflected wave which, when added vectorially to the first term of eq 12 , comprises the space wave. The third term of eq 12 represents the surface wave associated with the $\mathrm{r}-\mathrm{f}$ currents actually flowing in the ground.

The total field given by eq 12 is usually referred to as the ground wave. Propagation via the 


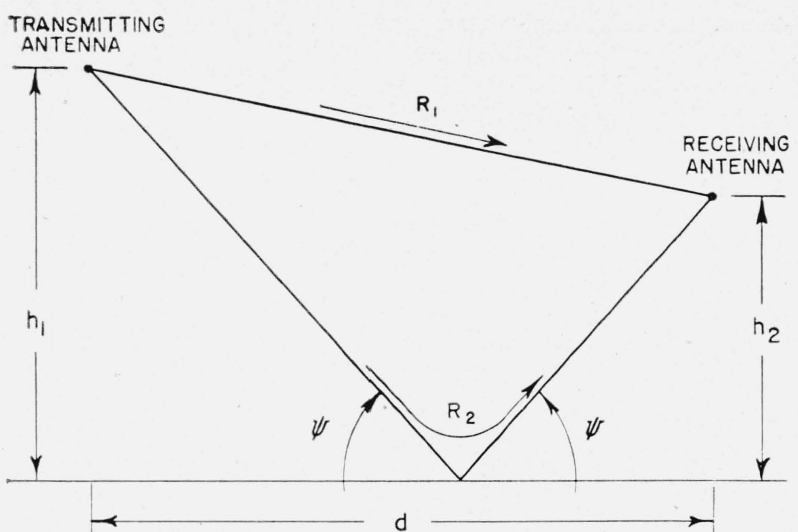

FigURE 10. Ray-path diagram showing: (a) direct ray along $R_{1}$; (b) ground-reflected ray along $R_{2}$ making an angle $\psi=\tan ^{-1}\left(h_{1}+h_{2}\right) / d$ with the earth.

Heights of the transmitting and receiving antennas are $h_{1}$ and $h_{2}$, respectively, and $d$ is the horizontal distance of separation.

ionosphere or troposphere, which is subject to changing solar or meteorological conditions is being ignored here.

The magnitude of the surface wave is usually negligibly small for horizontally polarized transmission at these frequencies [11], and in the remaining formulas used herein we shall neglect its presence entirely.

The complex reflection coefficient of the ground, $\Gamma$, may be expressed in terms of the angle $\psi=$ $\tan ^{-1}\left(h_{1}+h_{2}\right) / d$ and a complex dielectric constant $\boldsymbol{\epsilon}_{0}$, as follows, for horizontal polarization [12],

$$
\Gamma=\frac{\sin \psi-\sqrt{\epsilon_{0}-\cos ^{2} \psi}}{\sin \psi+\sqrt{\epsilon_{0}-\cos ^{2} \psi}}
$$

where

$$
\begin{aligned}
& \boldsymbol{\epsilon}_{0} \equiv \boldsymbol{\epsilon}_{r}\left(1-j \frac{\sigma}{\epsilon \omega}\right)=\epsilon_{r}-j 60 \lambda \sigma \\
& \boldsymbol{\epsilon}_{r}=\text { relative dielectric constant of the ground } \\
& \quad \text { (referred to free space as unity) } \\
& \boldsymbol{\epsilon} \equiv \boldsymbol{\epsilon}_{r} \epsilon_{r}, \text { where } \epsilon_{v} \text { is the permittivity of evac- } \\
& \quad \text { uated free space } \\
& \boldsymbol{\epsilon}_{v} \cong \frac{1}{36 \pi} \times 10^{-9} \text { farad per meter } \\
& \sigma=\text { ground conductivity, in mhos per meter } \\
& \omega=2 \pi f \\
& \lambda=\text { wavelength, in meters } \\
& f=\text { frequency, in cycles per second }
\end{aligned}
$$

Values of the magnitude of eq 13, $\rho$, are shown versus $\tan \psi=\left(h_{1}+h_{2}\right) / d$ in figure 11 for various values of the relative dielectric constant, $\boldsymbol{\epsilon}_{\tau}$, for low-loss dielectrics $(\sigma / \epsilon \omega \ll 1)$. Many types of ground may be treated as low-loss dielectrics over a large portion of the VHF band as far as their reflecting properties are concerned. This is particularly true at the higher frequencies above 50 or $75 \mathrm{Mc}$.

In this case the phase shift on reflection $\phi$, is very nearly $180^{\circ}$ for horizontal polarization, so that the value- of the reflection coefficient is given approximately by

$$
\Gamma \cong-\rho .
$$

Ignoring the surface wave altogether, the magnitude of eq 12 may be written (in volts per meter)

$$
|E| \cong \frac{60 \pi l_{H} I}{\lambda}\left|\frac{1}{R_{1}}+\frac{\Gamma e^{-j k\left(R_{2}-R_{1}\right)}}{R_{2}}\right| .
$$

Equation 15 may be expanded without further approximation, giving (in volts per meter)

$$
\begin{aligned}
& |E| \cong \frac{60 \pi l_{H} I}{\lambda} \\
& \left\{\left(\frac{1}{R_{1}}+\frac{\rho}{R_{2}}\right)^{2}-\frac{4 \rho}{R_{1} R_{2}} \sin ^{2}\left[\frac{k\left(R_{2}-R_{1}\right)+\phi}{2}\right]\right\}^{\frac{1}{2}},
\end{aligned}
$$

where $\rho$ is the magnitude of the reflection coefficient, $\Gamma$, and $\phi$ is its phase lag in radians. Equation 16 is in a form somewhat more suitable for computation, since no loss of accuracy is involved as a result of the taking of small differences between large computed quantities.

In case the approximation given by eq 14 applies, eq 16 may be written (in volts per meter)

$$
|E| \cong \frac{60 \pi l_{H} I}{\lambda}\left\{\left(\frac{1}{R_{1}}-\frac{\rho}{R_{2}}\right)^{2}+\frac{4 \rho}{R_{1} R_{2}} \sin ^{2}\left[\frac{k\left(R_{2}-R_{1}\right)}{2}\right]\right\}^{1 / 2}
$$

The quantity $\left[\left(1 / R_{1}\right)-\left(\rho / R_{2}\right)\right]^{2}$ within the radical becomes negligibly small when $d \gg\left(h_{1}+h_{2}\right)$, since $R_{1} \rightarrow R_{2} \rightarrow d, \rho \rightarrow 1$, and $R_{2}-R_{1} \cong 2 h_{1} h_{2} / d$. Under these conditions, eq 17 becomes (in volts per meter)

$$
|E| \cong \frac{120 \pi l_{H} I}{\lambda d}\left|\sin \left(\frac{2 \pi h_{1} h_{2}}{\lambda d}\right)\right|
$$

Equation 18 is obviously not valid in the vicinity of the zeros of the sine term, since the first term of eq 17 is not then negligible. Therefore use of eq 18 should preferably be restricted to values of $h_{1} h_{2} / \lambda d<0.5$. If either the wavelength, $\lambda$, or the distance, $d$, is increased, eq 18 will reach its last maximum value when $h_{1} h_{2} / \lambda d=0.3229 \ldots$. after which it will steadily decrease. If the antenna 


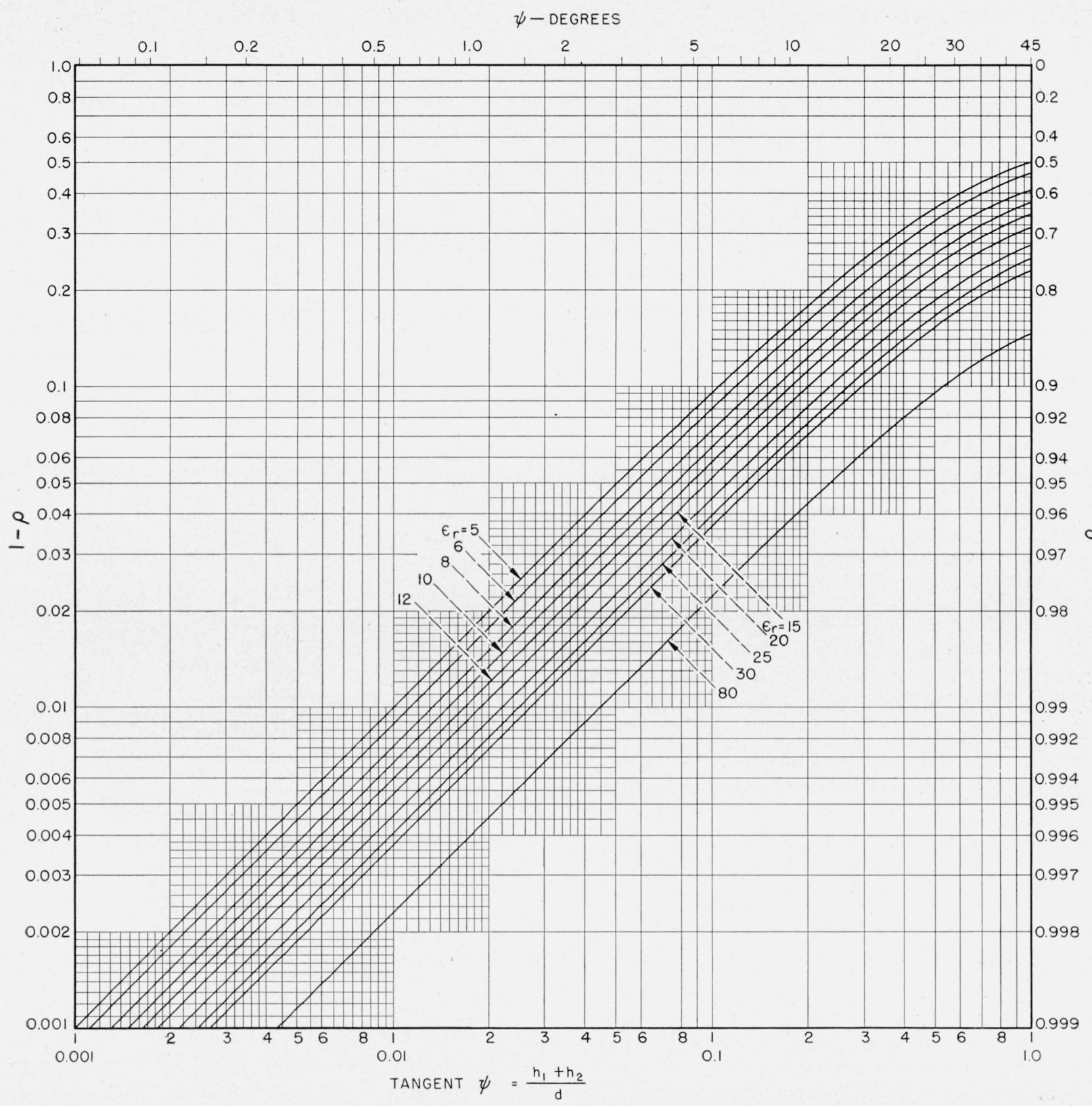

FIGURE 11. Variation of the magitude, $\rho$, of the complex plane-wave reflection coefficient, $\Gamma$, versus tan $\psi=\left(h_{1}+h_{2}\right) / d$ for several values of relative dielectric constant, $\epsilon_{r}$.

The curves apply to low-loss dielectries, $(\sigma / \epsilon \omega \ll 1)$, and horizontal polarization only and were determined from eq 13 .

heights $h_{1}$ or $h_{2}$ above are varied, this maximum will occur when $h_{1} h_{2} / \lambda d=0.25$.

When $2 \pi h_{1} h_{2} / \lambda d \leqq 1 / 4$ the field intensity is given (in volts per meter) to within 1 percent by

$$
|E| \cong \frac{240 \pi^{2} l_{H} I}{d^{2}}\left(\frac{h_{1} h_{2}}{\lambda^{2}}\right)
$$

since $\sin \theta \cong \theta$ to within the stated accuracy for $\theta \leq 1 / 4$ radian.

When attempting to establish values of standard field intensity, however, one should rely on eq 16 or 17 until the assumptions applying to eq 18 and 19 are found to be fully justified. 


\section{Description of the Standard Transmitting Antenna}

The transmitting antenna used was similar in many respects to the standard receiving antenna described in section II. A horizontal half-wave dipole was used shortened 4 percent for selfresonant operation at $100.0 \mathrm{Mc}$. Dural tubing $3 / 16$ in. in diameter was used, giving a ratio $2 l / a \cong 600$ and an average characteristic impedance $K_{0}=120\left[\log _{e}(2 l / a)-1\right] \cong 650$ ohms.

The current at the center of the antenna was measured by means of a previously calibrated VHF vacuum thermocouple and a section of balanced twin-lead transmission line one-half wavelength long. The antenna and thermocouple terminated the transmission line at opposite ends, and a balanced feed-line from the $\mathrm{r}-\mathrm{f}$ generator was tapped in at the midpoint. Thus the currents flowing at the opposite ends of the half-wave line were identical in value. The magnitude of the antenna current was thus indicated by the thermocouple $\lambda / 2$ distant and was entirely independent of the magnitude and phase angle of the terminations, namely, the antenna input impedance, and the impedance of the thermocouple heater. A broadband balun [7] was used to transform the unbalanced output of the r-f generator to the balanced transmission system. A block diagram of the equipment and its arrangement are shown in figure 12. The thermocouple was calibrated in a similar manner to that described earlier for the receiving antenna voltmeter, and was found to have a frequency error of 0.5 percent at $100 \mathrm{Mc}$.

\section{Propagation Tests}

\section{General}

In order to determine the probable accuracy of the VHF field-intensity determinations made by the standard-antenna and standard-field methods previously described, actual propagation tests were made at a frequency of $100.0 \mathrm{Mc}$, using horizontal polarization only. The purpose was to actually intercompare values of field-intensity determined with the two standards.

For instance, a predetermined value of field intensity was established by means of the standardfield method, and simultaneously measured using the standard (receiving)-antenna method, and the results compared. However, instead of relying on

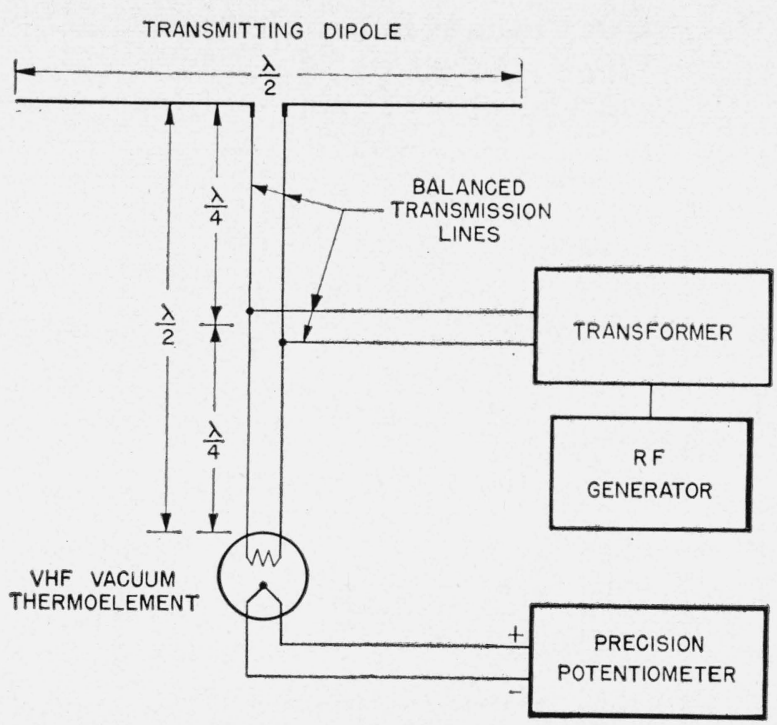

FIGURE 12. Diagram showing the method used for determining the $r-f$ current at the center of the transmitting dipole.

isolated pairs of such measurements to determine the probable accuracy of the two methods, more or less complete propagation runs were made involving some 15 or 20 pairs of determinations in each, in order to determine the trend with varying antenna height or distance of separation. In this manner it was possible to determine the range of antenna heights and distances of separation between the antennas over which the previous assumptions applying to the earth were valid, and to what extent we were dealing with a uniform field.

Three general types of tests were made as follows:

(a) Vertical-incidence standing-wave measurements, in which the standard field beneath the horizontal transmitting antenna was probed vertically using the standard (receiving) antenna.

(b) Variable-height runs, in which the heights of the transmitting and receiving antennas were varied separately for a fixed horizontal distance of separation.

(c) Variable-distance runs, in which the horizontal distance between the transmitting and receiving antennas was varied for several fixed antenna heights.

In order to conduct these tests it was necessary to select a special site. The usual requirements specify that the location be relatively flat and free of such reflecting objects as trees, buildings, wires, 
etc. in order to approximate the conditions assumed to exist in deriving the standard field formulas in section III.

A site of approximately 100 acres was made available at the Beltsville, Md. Airport for these tests. The land had been cleared and graded and met the above requirements. Interference from aircraft was negligible, inasmuch as the airport was officially closed and used only for emergency landings.

One other requirement, dictated by the lack of selectivity in the standard antenna, made it necessary to conduct such tests at a location where the field intensity of frequency-modulation and television broadcast stations was negligible compared to the standard field being used. Such interference was further minimized (since the test site was sufficiently free of reflecting objects) by properly orienting the two antennas. This was possible in our case since the interfering stations, some 20 miles distant, were all approximately in the same direction from the test site.

\section{Vertical-Incidence Standing-Wave Measure- ments}

This run was originally intended to be used for determining the reflection coefficient and hence the apparent dielectric constant, and conductivity of the ground at the test site. However, it was also found to be useful later in determining the effective length of the transmitting and receiving antennas, as will be shown.

For this test the horizontal transmitting dipole was located at the top of an all-wood ladder-mast at a fixed height $h_{1}=9.27 \mathrm{~m}$. The balanced unshielded 75-ohm r-f transmission line ran at right angles to the antenna and approximately $10 \mathrm{deg}$ below the horizontal. The unshielded balanced d-c output lead from the thermocouple paralleled the $\mathrm{r}-\mathrm{f}$ line and was spaced approximately $3 \mathrm{ft}$ with negligible $r-f$ pickup. The $r-f$ power generator and associated equipment were located on the ground approximately $200 \mathrm{ft}$ distant. The transmitting-antenna current was continuously monitored by an operator at the generator and was held constant to within \pm 0.1 percent. The d-c output of the thermocouple was measured on a portable precision potentiometer. The probable accuracy of the antenna-current measurement was about 1 percent. The frequency of the $r-f$ generator was also continuously monitored by means of a calibrated heterodyne frequency meter, and held to $100.0 \mathrm{Mc} \pm 0.1$ percent.

The standard (receiving) antenna (oriented parallel to the transmitting antenna), was mounted on a movable carriage on the same mast, and was readily adjustable in height by means of a continuous rope passing through nonmetallic pulleys at the base and top of the mast. Certain predetermined heights were marked directly on the side of the mast and read by a second operator located at some distance off the end of the antenna in a null. The actual height of the antenna above ground could be thus measured to better than 0.5 $\mathrm{cm}$. The balanced d-c output of the receiving antenna crystal-rectifier was carried over rubbercovered lamp cord, which passed vertically down the mast to the ground. From this point the d-c line went directly to the operator's position where the voltage was measured on the precision slidewire potentiometer previously mentioned. Tests were made for r-f pickup on the d-c line, and in the resistance-capacitance filter at the antenna, with the antenna rods removed, but was found to be negligible even with the d-c line running the entire height of the mast.

Tests were made for stray radiation from the transmitting system caused by any slight unbalance in the feed line to the antenna or by leakage. The transmitting antenna rods were removed and a 65-ohm high-frequency resistor substituted to terminate the transmission line. With the normal r-f current of $0.1 \mathrm{amp}$ flowing, the resulting field was too small to be measured for any height of the receiving antenna. The vertical component of field existing at the receiving antenna was more than $40 \mathrm{db}$ below the main field, with the horizontal transmitting antenna excited.

Returning to the description of the verticalincidence measurements, the resulting interference between the direct and ground-reflected waves, set up a standing-wave in the space beneath the transmitting antenna, as shown in figure 13. The standard (receiving) antenna was moved vertically up or down the mast to measure the resulting value of field intensity. The reflection coefficient was accurately determined from the standingwave ratio, as will be shown. Once this was known, the expressions for the field intensity for the standard-antenna and standard-field methods, eq 2 and 15, were equated and solved for the remaining unknown, the effective length of the 
antennas. This was deemed permissible since both antennas were physically identical.

The field intensity as determined by the standard-field method was obtained by substituting $R_{1}=h_{1}-h_{2}, R_{2}=h_{1}+h_{2}, d=0$ in eq 15 , giving (in volts per meter)

$$
|E| \cong \frac{60 \pi l_{H} I}{\lambda}\left|\frac{1}{h_{1}-h_{2}}+\frac{\Gamma e^{-j 2 k h_{2}}}{h_{1}+h_{2}}\right| .
$$

If $h_{1} \gg h_{2}$, the value of field intensity given by eq 20 will have a maximum value if $\left(2 k h_{2}+\phi\right)=2 n \pi$, and a minimum value if $\left(2 k h_{2}+\phi\right)=(2 n-1) \pi$, where $n=1,2,3, \ldots$. The magnitude of the ratio of the maximum to minimum values of field intensity is then

$$
S W R=\left|\frac{E_{\max }}{E_{\mathrm{min}}^{\prime}}\right| \simeq \frac{\frac{1}{h_{1}-h_{2 \max }}+\frac{\rho}{h_{1}+h_{2 \max }}}{\frac{1}{h_{1}-h_{2 \min }}-\frac{\rho}{h_{1}+h_{2 \min }}} .
$$

The magnitude, $\rho$, of the complex reflection coefficient, $\Gamma$, of the ground may be determined from the SWR and the heights, $h_{2}$, of the receiving antenna corresponding to the maximum and the minimum values of field intensity measured plus the height of the transmitting antenna, thus

$$
\rho \cong \frac{\frac{S W R}{h_{1}-h_{2 \min }}-\frac{1}{h_{1}-h_{2 \max }} .}{\frac{S W R}{h_{1}+h_{2 \min }}+\frac{1}{h_{1}+h_{2 \max }}}
$$

The angle of phase lag, $\phi$, of the reflection coefficient may be determined from the shift in the positions of the maxima or minima from the positions corresponding to $\phi=0$. For the maxima this gives

$$
\phi=2\left[\frac{2 \pi h_{2 \max }}{\lambda}-(n-1) \pi\right],
$$

where $n=1,2,3, \ldots$ is the number of the maximum used to calculate $\phi$, counting from the ground upward.

In using the minima to determine $\phi$, the expression is

$$
\phi=2\left[\frac{(2 n-1) \pi}{2}-\frac{2 \pi h_{2 \min }}{\lambda}\right],
$$

$h_{2 \min }$ and $h_{2 \max }$ are the actual heights, in meters, at which the particular minimum or maximum occurs.

In determining the value of the reflection co-

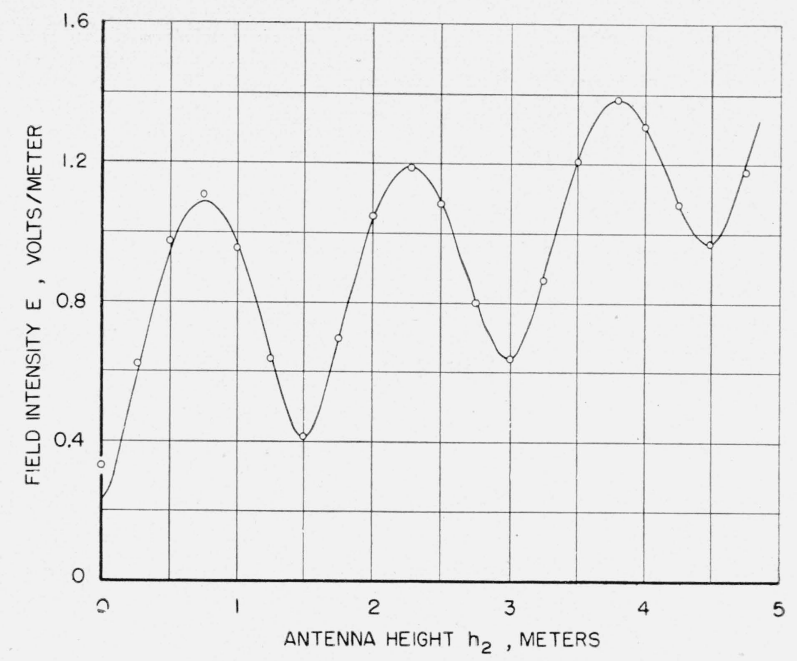

FIGURE 13. Variation of the observed and calculated values of electric field intensity, $|E|$ in volts per meter, versus the height, $h_{2}$, in meters, of the standard receiving antenna.

The transmitting antenna was located above and parallel to the receiving antenna at a fixed height, $h_{1}=9.27 \mathrm{~m}, d=0, f=100.0 \mathrm{Mc}, I=0.100 \mathrm{amp}$.

$\bigcirc$, observed, -

efficient of the ground at the test site, the heights, $h_{2}$, of the various maxima and minima were carefully measured. It was found possible to determine these to within $\pm 1.0 \mathrm{~cm}$. To within this accuracy the positions corresponded to a phase shift, $\phi$, on reflection of $180 \mathrm{deg}$. The possible error in determining $\phi$ thus amounts to approximately $2 \mathrm{deg}$. It is interesting to note that the calculated phase shift, $\phi$, for average ground $\left(\epsilon_{r}=\right.$ $15, \sigma=5 \times 10^{-3}$ mho per meter) at $100 \mathrm{Mc}$ is very nearly $179 \mathrm{deg}$ at normal incidence. An error of $2 \mathrm{deg}$ in determining $\phi$ usually has a negligible effect on the magnitude, $\rho$, of the reflection coefficient, and on the resulting value of field intensity calculated.

Although the standing-wave method cannot be used to determine even approximately the ground conductivity at these frequencies, such information is usually not required if the reflection coefficient can be accurately determined.

An example will be given of the method of determining the magnitude, $\rho$, of the reflection coefficient, and the dielectric constant, $\epsilon_{r}$, of the ground from the observed data presented in figure 13. Three different values of standing-wave ratio were determined from the three maxima and the three succeeding minima. The minimum occurring at the ground $\left(h_{2}=0\right)$ was ignored because of its questionable value. 
The value of $\rho$ corresponding to each SWR was then determined from eq 22 , and the resulting value of the relative dielectric constant $\epsilon_{r}$ determined from the relation

$$
\epsilon_{r}=\left(\frac{1+\rho}{1-\rho}\right)^{2}
$$

which was obtained from eq 13 by substituting $\psi=\pi / 2$, and $\Gamma=-\rho$ and solving for $\epsilon_{r},(\sigma / \epsilon \omega \ll 1)$. The results are given in table 1 .

TABLE 1. Values of $\rho$ and $\epsilon_{r}$, as determined from standingwave measurements

\begin{tabular}{|c|r|r|}
\hline \multicolumn{1}{|c|}{$S W R$} & \multicolumn{1}{|c|}{$\rho$} & \multicolumn{1}{c|}{$\epsilon_{r}$} \\
\cline { 1 - 2 } & & \\
\cline { 2 - 3 } 2.67 & 0.648 & 21.9 \\
1.85 & .635 & 20.1 \\
1.42 & .636 & 20.2 \\
\cline { 2 - 3 } Average_.. & .640 & 20.7 \\
\hline
\end{tabular}

The values of $\rho$ and $\epsilon_{\tau}$ measured are determined largely by the moisture content of the ground. Consequently, their values fluctuate somewhat from day to day with changing moisture content. Four values of $\epsilon_{r}$ obtained in a similar manner over a period of a few months are shown in figure 14, together with values of daily rainfall. It can be seen that the variation in the dielectric constant is quite large. Normally, this is not of much concern to the propagationist, who is usually interested in grazing incidence, but it is apt to affect the accuracy of standard-field measurements at very short distances if the incident angle is large. Near the surface the moisture content and consequently the ground constants themselves may vary with depth. Since the skin depth in turn varies with frequency and angle of incidence (among other factors), the (apparent) values determined for these constants may also depend on these parameters.

If desired, the reflection coefficient of the ground for vertical incidence may be calculated from eq 13 upon substituting $\psi=\pi / 2$, giving

$$
\Gamma=\frac{1-\sqrt{\epsilon_{r}\left(1-j \frac{\sigma}{\epsilon \omega}\right)}}{1+\sqrt{\epsilon_{r}\left(1-j \frac{\sigma}{\epsilon \omega}\right)}} .
$$

Values of the magnitude of eq 26 are plotted versus $\epsilon_{T}$ in figure 15 for low-loss dielectrics, $(\sigma / \epsilon \omega \ll 1)$, remembering that $\Gamma \cong-\rho$ for this condition.

Determining the value of the reflection coefficient, $\Gamma$, of the ground from the observed data of figure 13 actually required only relative values of the measured field intensity. With the value of $\Gamma$ now accurately determined, the only remaining unknown in eq 20 is the effective length, $l_{H}$, of the transmitting antenna. Since the standard transmitting and receiving antennas used are geometrically identical, the assumption is made that their effective lengths are equal. There has been some indication in the works of King [13] that such is not excatly the case, and further that the value of $l_{H}$ is also somewhat dependent upon the antenna termination. However, it is shown that for self-resonant antennas these differences are small. Shelkunoff does not elaborate on these points, but our experimental values of

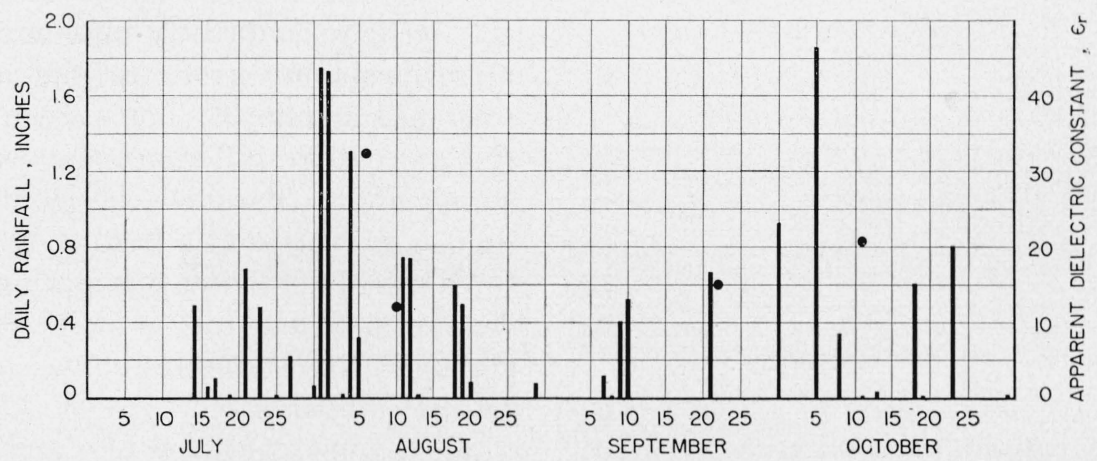

FIGURE 14. Chart showing four measured values of the apparent relative dielectric constant, $\epsilon_{r}$, of the ground at one particular site, as affected by rainfall. $f=100.0 \mathrm{Mc}$.

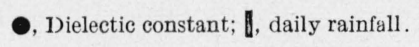


effective length agree well with those calculated from his analysis of cylindrical antennas, as will be shown. The value of the effective length, $l_{H}$, may be determined by equating eq 2 and 20, and solving for $l_{H}$.

The data shown in figure 13 provide 19 separate determinations of $l_{H}$ (ignoring the observed value corresponding to $h_{2}=0$ because of its questionable value). The average of these gives a measured value of $l_{H}=0.960 \mathrm{~m}$ for the self-resonant antennas used.

Figure 16 shows the percentage difference between each pair of determinations as presented in figure 13 , versus the height, $h_{2}$, of the receiving antenna. In this information is included experimental error, differences caused by changes in the receiving antenna current distribution due to the presence of the ground, and differences caused by the proximity of the transmitting and receiving antennas, of which the latter should be small in view of the fact that the receiving antenna was operated open-circuited. These differences, in general, are less than 3 percent, and give a good indication of the agreement possible between the standard-antenna and standard-field methods at least for the case of normal incidence where the reflection coefficient of the ground could be accurately determined.

It is also indicated that any varying effect (with antenna height) of the presence of the ground on

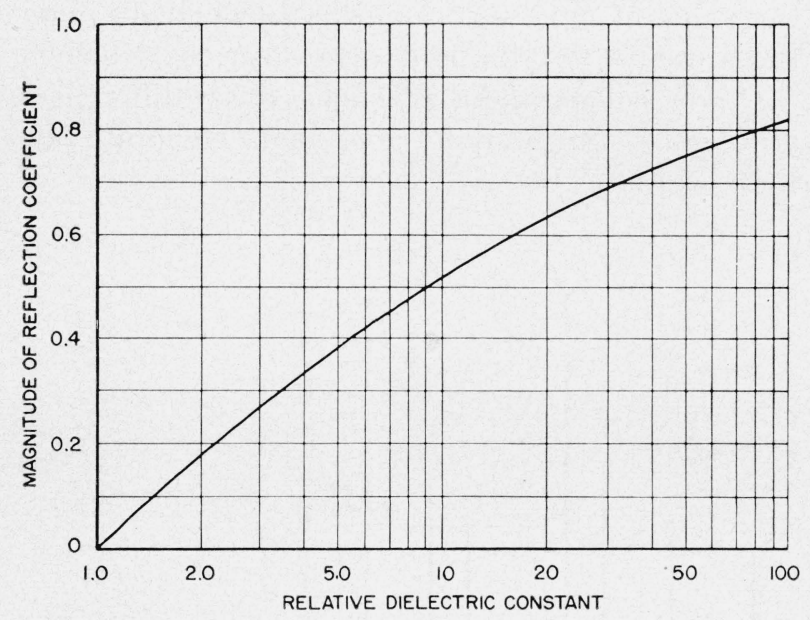

Figure 15. Calculated variation of the magnitude, $\rho$, of the complex plane-wave reflection coefficient of the ground $\Gamma$, versus the relative dielectric constant, $\epsilon_{r}$, for vertical incidence, $\psi=\pi / 2$.

The curve applies to low-loss dielectrics only $(\sigma / \epsilon \omega \ll 1)$, and was determined from eq 26.

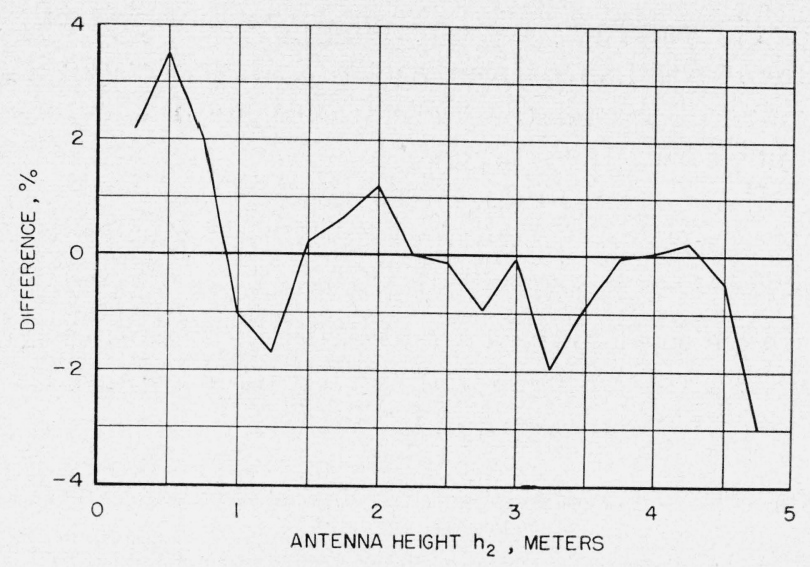

Figure 16. Variation of the percentage difference between the observed and calculated values of field intensity of figure 13, with height, $h_{2}$, of the receiving antenna.

the receiving antenna current distribution and hence upon its effective length is negligible, at least for the present purpose for antenna heights in excess of 0.1 wavelength.

The calculated value of the effective length is obtained from the product of the following values: (a) the classical value of effective length for a half-wave dipole given by eq 4; (b) the percentage shortening for self-resonant operation from figure 3 ; and (c) the percentage increase for cylindrical antennas from figure 2. The resulting value is $l_{H} \cong \lambda / \pi \times 0.96 \times 1.06 \cong 0.97 \mathrm{~m}$ for operation at 100 $\mathrm{Mc}$, and agrees to within about 1 percent with the above measured value.

\section{Variable Height Runs}

The purpose of the tests described in this section was to determine the probable measurement error existing in the two field-intensity standards under discussion. Determinations made by the two standards were directly intercompared, the maximum probable error (in the absence of other reference standards) being evaluated as one-half the percentage difference between the two measurements. Parallel horizontal self-resonant dipoles were used at a frequency of $100.0 \mathrm{Mc}$.

Values of field intensity established by means of the standard-field method were measured using the standard (receiving)-antenna method and the results compared.

These runs consisted of varying separately the heights of either the receiving or transmitting antennas for a fixed height of the other antenna, and for a fixed distance of separation. 


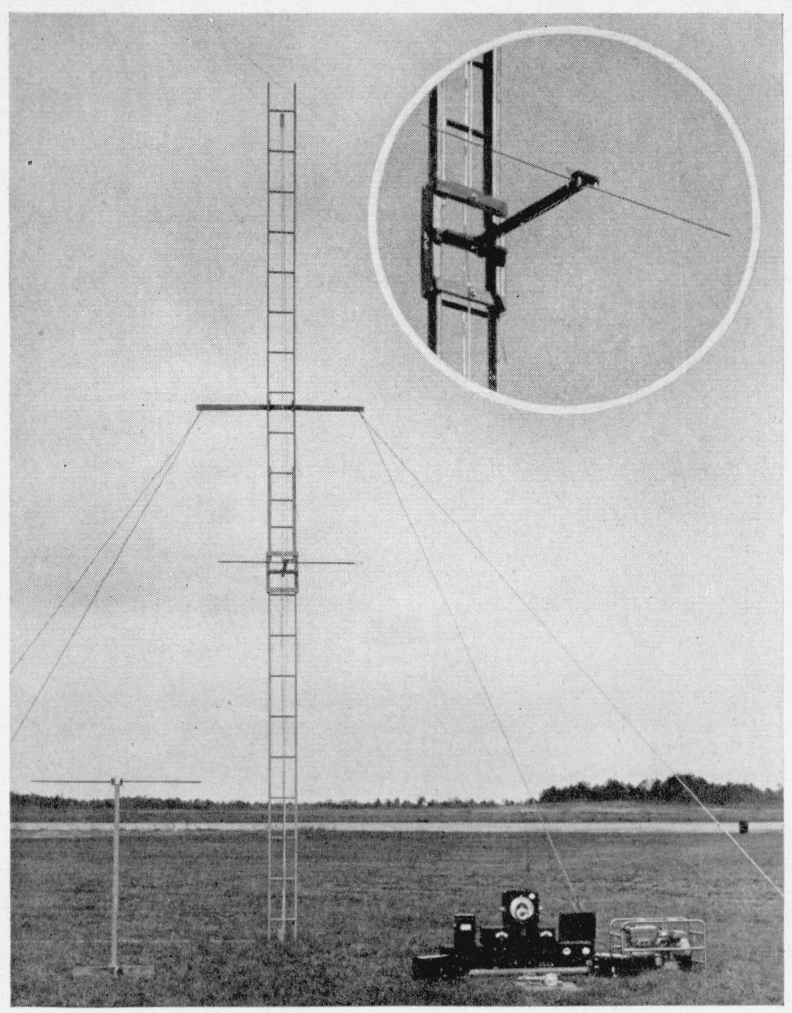

Figure 17. View of the various pieces of equipment used in obtaining the measured data of figures 13, 14, 18, 19, and 20.

In the background and insert are shown the ladder mast and carriage for the receiving dipole. The transmitting antenna, $r-f$ generating equipment, and an engine-driven alternator are shown in the foreground. The location is the Beltsville (Md.) Airport.

In the first two runs the receiving antenna was varied in height, $h_{2}$, from 0.265 to $9.0 \mathrm{~m}$, using the ladder-mast assembly shown in figure 17 . The heights, $h_{1}$, of the transmitting antenna were 1.58 and $3.05 \mathrm{~m}$, respectively, and the horizontal distance of separation, $d$, was $30.5 \mathrm{~m}$. The measured value of the effective length of the antennas determined in the standing-wave test was used, namely, $l_{H} \cong 0.96 \mathrm{~m}$.

The results are shown in figure 18. In determining the field by the standard-antenna method, eq 2 was used (in volts per meter)

$$
|E|=\frac{V_{o c}}{l_{H}},
$$

where $V_{o c}$ is the measured value of open-circuit voltage.

The value of field determined by the standardfield method was obtained (in volts per meter) from eq 17 ,

Field-Intensity Standards
$|E| \cong \frac{60 \pi l_{H} I}{\lambda}\left\{\left(\frac{1}{R_{1}}-\frac{\rho}{R_{2}}\right)^{2}+\frac{r 4 \rho}{R_{1} R_{2}} \sin ^{2}\left[\frac{k\left(R_{2}-R_{1}\right)}{2}\right]\right\}^{1 / 2}$,

where

$$
\begin{aligned}
l_{H} & =0.96 \mathrm{~m} \\
I & =0.100 \mathrm{amp} \\
\lambda & =3.00 \mathrm{~m} \\
k & =2 \pi / \lambda \\
R_{1} & =\left[\left(h_{1}-h_{2}\right)^{2}+d^{2}\right]^{1 / 2} \\
R_{2} & =\left[\left(h_{1}+h_{2}\right)^{2}+d^{2}\right]^{1 / 2} .
\end{aligned}
$$

Values of the magnitude, $\rho$, of the reflection coefficient were determined by taking the magnitude of eq 13, assuming low-loss dielectrics, $(\sigma / \epsilon \omega \ll 1)$. Values of $\rho$ may also be taken from figure 11 .

It was not found practical to measure the dielectric constant of the ground before each run because of the special setup required and the time consumed. Previous measurements showed that on the average the dielectric constant had a value around 15, except after a rain or after a prolonged dry spell. This value of $\epsilon_{r}$ was therefore used in calculating $\rho$ from eq 13 .

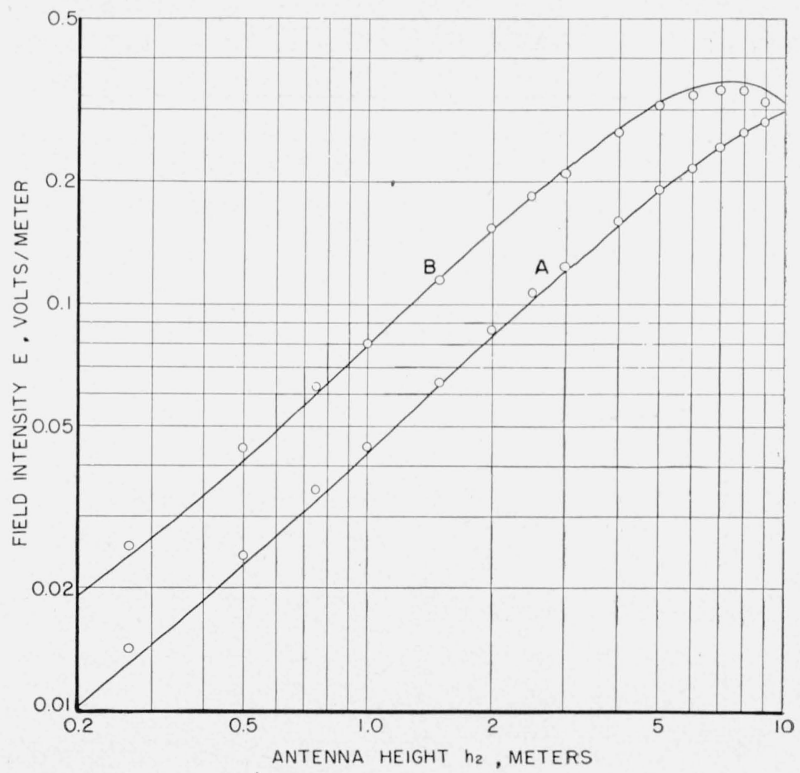

Figure 18. Variation of the magnitude of the electric component of field intensity, $|E|$ in volts per meter as determined by the standard-antenna and standard-field methods vs the height, $h_{2}$ in meters, of the standard receiving antenna.

Fixed heights $h_{1}=1.58 \mathrm{~m}(\mathrm{~A})$, and $3.05 \mathrm{~m}(\mathrm{~B})$ of the transmitting antenna were used, and $d=30.5 \mathrm{~m}$. $f=100.0 \mathrm{Mc}, I=0.100 \mathrm{amp}, \epsilon_{\mathrm{r}}=15, \sigma=0$. Horizontal self-resonant half-wave dipoles were used having effective lengths of $0.960 \mathrm{~m}$. $\bigcirc$, Standard-antenna method; - , standard-field method. 
The diameter of the circles representing the standard-antenna data in figure 18 is approximately 5 percent of the value of field intensity. Thus the difference is 2.5 percent or less for those points that touch the solid curves. Over most of the range of heights, $h_{2}$, the agreement is within about 5 percent.

A similar run is shown in figure 19, except that the height of the transmitting antenna was varied for a fixed height of the receiving antenna. The antenna current, $I$, was maintained constant at 0.100 amp. The other conditions are the same as those applying to figure 18 . The agreement between the two standards is about the same as in the two previous runs.

\section{Variable-Distance Runs}

In these last tests the field intensity versus the horizontal distance of separation, $d$, was determined for fixed heights of the transmitting and receiving antennas.

The receiving antenna was maintained at a fixed height $h_{2}=3.05 \mathrm{~m}$, and the distance, $d$, varied from 6 to $60 \mathrm{~m}$ for each of two heights of

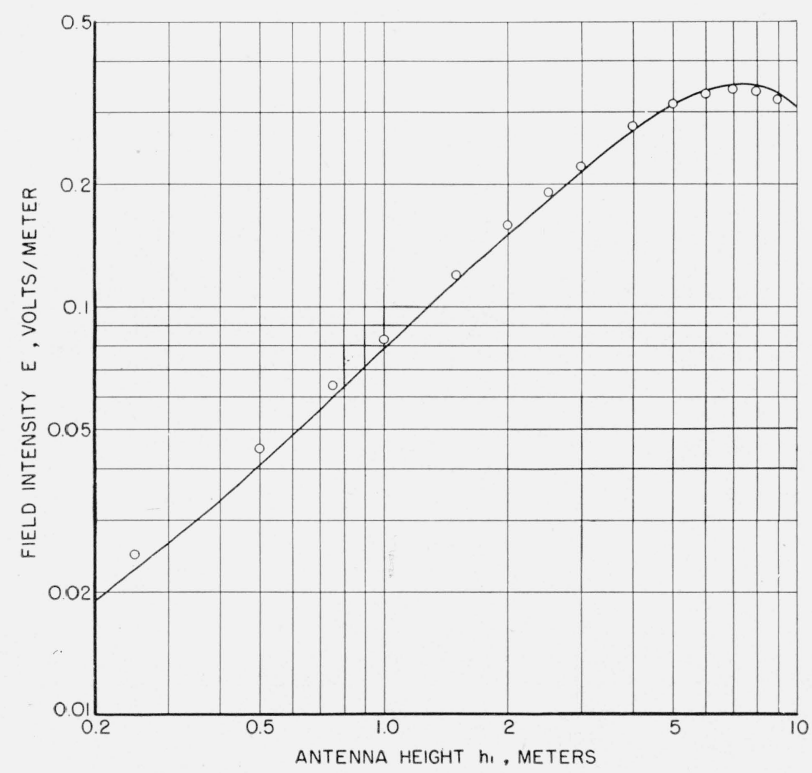

FIG URE 19. Variation of the magnitude of the electric component of field intensity, $|E|$ in volts per meter, as determined by the Standard-Antenna and Standard-Field methods versus the height, $h_{1}$ in meters, of the transmitting antenna.

A fixed height, $h_{2}=3.05 \mathrm{~m}$, of the receiving antenna was used. The remaining conditions are identical to those applying to figure $18 . \bigcirc$, Standardantenna method; -, standard-field method.

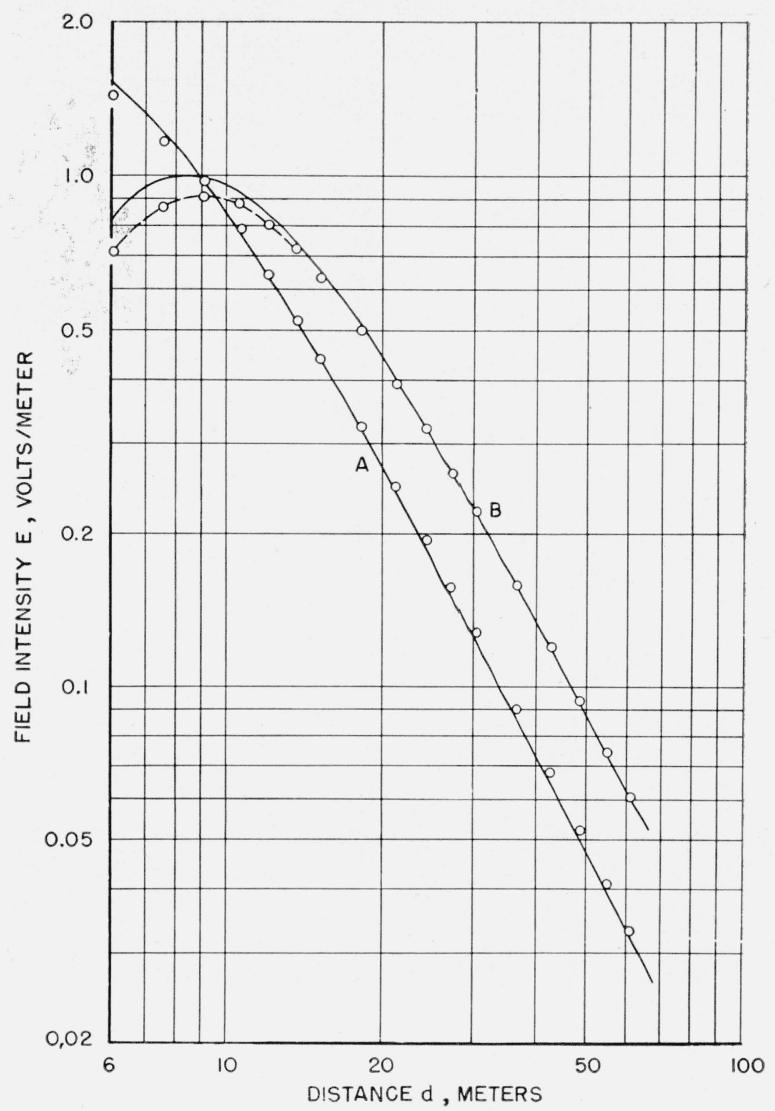

FIGURE 20. Variation of the magnitude of the electric component of field intensity, $|E|$ in volts per meter, as determined by the Standard-Antenna and Standard-Field methods versus the horizontal distance of separation, $d$ in meters, between the antennas.

Fixed heights of the transmitting antenna $h_{1}=1.58 \mathrm{~m}(\mathrm{~A})$, and $3.05 \mathrm{~m}(\mathrm{~B})$, and a fixed height of the receiving antenna, $h_{2}=3.05 \mathrm{~m}$ were used. The remaining conditions are identical to those applying to figure 18 . $\bigcirc$, Standard-antenna method; - , standard-field method.

the transmitting antenna $h_{1}=1.58$ and $3.05 \mathrm{~m}$, respectively.

Horizontal self-resonant half-wave dipoles were used as before. The field intensity was determined for various distances of separation using both the standard-antenna and standard-field methods. The remaining conditions are the same as those in the previous section. The results are shown in figure 20.

The values of field intensity determined by both the standard-antenna and standard-field methods (figs. 13, 18, 19, and 20) seem to be in fairly good agreement over most of the range of antenna heights or distance of separation involved. It is reasonable to assume that the effective length of both antennas remains fixed throughout the entire 
range. The larger disagreements noted are therefore probably a result of errors in calculating the standard field because of the accuracy with which the actual ground constants are known for the various incident angles involved.

\section{Conclusions}

A description has been given of two standard methods of establishing known values of VHF field intensity used at the National Bureau of Standards for the calibration of commercial fieldintensity meters. Methods and techniques used to calibrate and verify the accuracy of the standards were described. Values of standard field intensity can be established in the range from 20 $\mathrm{mv} / \mathrm{m}$ to $1 \mathrm{v} / \mathrm{m}$ at $100 \mathrm{Mc}$. This range can be considerably increased by varying the transmitting-antenna current in known amounts by means of the standard attenuator previously mentioned. The propagation tests described indicate that the probable accuracy of the standards is about 5 percent under most of the conditions used.

The limitations in the use of the standards lie in the somewhat restricted range of field intensity available, the temperature effect of the crystal rectifier, and for some uses the lack of selectivity in the standard receiving antenna. However, for the purposes reported here and under the conditions used these factors presented little difficulty.

The initial work described in this report was confined to the use of horizontal polarization at a frequency of $100 \mathrm{Mc}$. Further tests are in progress to verify the accuracy of these calibration standards at frequencies up to $300 \mathrm{Mc}$ under conditions normally used in the field. The additional use of vertical as well as circular polarization will be included in this program.

Under most conditions normally employed for calibrating VHF field-intensity meters, the value of the antenna constant ${ }^{6}$ as determined by means of horizontally polarized transmission would probably be substantially the same if vertical polarization were employed. This would be approximately true provided the antenna were immersed in a uniform field in both instances, and that its

${ }^{6}$ The antenna constant may be defined as $K=1 / \eta l_{H}$, where $\eta$ is the voltagetransfer ratio defined by eq 1 . height above ground was greater than about a half wavelength. The antenna constant can be shown to be influenced by the effect of the presence of the ground on the current distribution along the antenna, as well as on its input impedance. Although, these effects are different for horizontal polarization than for vertical, they are probably relatively small for antenna heights above approximately a half wavelength.

Results presented herein indicate that for the case of horizontal polarization any possible effect of the ground on the antenna current distribution probably results in less than about a 2-percent change in the antenna constant for antenna heights greater than 0.1 wavelength.

The effect of the presence of the ground on the antenna constant as caused by changes in the antenna input impedance has been shown to be less than about 5 percent for antenna heights greater than 0.65 wavelength above average ground $\left(\epsilon_{r}=15, \sigma=5 \times 10^{-3} \mathrm{mho} / \mathrm{m}\right)$ [14].

In the calibration of commercial VHF fieldintensity meters the accuracy of calibration as certified by this Bureau at the present time would probably be from twice to several times the accuracy of the standards described, depending upon the merits of the individual meter under test.

\section{Appendix. A Summary of Schelku- noff's Analysis of the Current Distribu- tion on a Thin Cylindrical Antenna}

Schelkunoff [1] obtained an approximate solution for the free-space current distribution on a hollow cylindrical antenna by analogy with a thin biconical antenna, together with the theory of the nonuniform transmission line.

The antenna region and the space beyond are considered as two multiple transmission lines or waveguides in tandem, each with its own distinct set of transmission modes.

In the antenna region (that space within a geometrical boundary sphere passing through the ends of the antenna) the field is composed of a principal or dominant mode plus a complementary set of an infinite number of higher order modes. The principal mode or wave, is that usually as sociated with a two-conductor transmission line. The complementary waves are generated in order to match the field (at the boundary sphere) to the field in the external region where no principal mode of transmission exists. Because of the nonexistence of physical conductors in the external region, the field consists solely of an infinite set of higher-order transmission modes. While these sets 
are distinct in their respective regions, they approach equality at the boundary, thus preserving the continuity of the field.

Associated with the principal and complementary waves in the antenna region are the principal current $I_{0}(r)$, and the complementary current flowing on the antenna given by

$$
\bar{I}(r)=I_{1}(r)+I_{3}(r)+I_{5}(r) \ldots,
$$

where the even-order modes vanish because of the symmetry involved.

At the ends of the hollow cylindrical doublet antenna $(r=l)$ the total current is essentially zero or

$$
I(l)=I_{0}(l)+\tilde{I}(l)=0 .
$$

Therefore,

$$
I_{0}(l)=-\ddot{I}(l) .
$$

Since it can be shown that the total voltage associated with any complementary wave is zero,

$$
V(r)=V_{0}(r)
$$

It can be seen that the presence of the complementary waves affect the amplitudes of the principal waves as would an impedance

$$
Z_{t}=\frac{V_{0}(l)}{I_{0}(l)}
$$

terminating the antenna.

As far as the principal waves are concerned, then, the antenna may be considered as a nonuniform transmission line terminated by an impedance $Z_{\mathrm{t}}=R_{\mathrm{t}}+j X_{\mathrm{t}}$. The real part, $R_{t}$, is the radiation resistance of outer space as seen from the ends of the antenna, and the imaginary part, $X_{t}$, represents the reactive field associated with the complementary waves. These two components represent the end effect usually associated with radiation from an antenna.

The current distribution on the antenna is therefore determined by the actual distribution of $L$ and $C$ along the antenna and by the presence of the complementary currents generated by reflection at the ends.

In a uniform parallel-wire transinission line, $L$ and $C$ per unit length, and consequently $Z_{0}$, are constant throughout. In a transmission line composed of a straight wire of length $2 l$, driven at the center, $L$ and $C$ vary continuously along its length. The potential difference between any two points equidistant from the center is simply the integral of the electric field intensity along a line of con- stant intensity terminating at the two points. The cylindrical doublet may be compared to such a nonuniform transmission line. $L$ and $C$ usually vary only slowly with $r$ in such a line, and by analogy to a thin biconical antenna with slowly varying cone angle, $\psi$, the characteristic impedance as a function of $r$ can be expressed approximately as

$$
K(r) \cong 120 \log _{e} \frac{2 r}{a}(r \gg a)
$$

The average characteristic impedance over length $l$ is

$$
K_{0} \cong \frac{1}{l} \int_{0}^{l} K(r) d r \cong 120\left(\log _{e} \frac{2 l}{a}-\right) .
$$

From the theory of the nonuniform transmission line, the relative distribution of the principal current is given for the case in which the variation in $K(r)$ is small

$$
\begin{aligned}
\frac{I(r)}{I(0)}= & {\left[\cos \beta r-\frac{M(r)}{K_{0}} \cos \beta r+\frac{N(r)}{K_{0}} \sin \beta r\right] } \\
& \frac{-j Z(0)}{K_{0}}\left[\sin \beta r+\frac{M(r)}{K_{0}} \sin \beta r+\frac{N(r)}{K_{0}} \cos \beta r\right],
\end{aligned}
$$

where

$$
\begin{aligned}
\beta= & 2 \pi / \lambda, \text { and } Z(0) \text { is the input impedance of } \\
& \text { the antenna } \\
M(r)= & \beta \int_{0}^{r}\left[K_{0}-K(r)\right] \sin 2 \beta r d r \\
= & 60(1-\cos 2 \beta r)\left[\log _{t}(l / r)-1\right]+60\left(\log _{e} 2 \beta r\right. \\
& -C i 2 \beta r+C) \\
N(r)= & \beta \int_{0}^{r}\left[K_{0}-K(r)\right] \cos 2 \beta r d r \\
= & 60\left(\log \frac{l}{r}-1\right) \sin 2 \beta r+60 S i 2 \beta r \\
S i(x) \equiv & \int_{0}^{x} \frac{\sin t}{t} d t \\
C i(x) \equiv & \int_{\infty}^{x} \frac{\cos t}{t} d t \\
C= & 0.5772 \ldots \text { (Euler's constant). }
\end{aligned}
$$

The relative complementary current for a biconical antenna and to a second approximation for any thin antenna is given by

$$
\frac{\bar{I}(r)}{I_{0}}=\frac{60}{K_{0}} \sum_{m=0}^{\infty} \frac{4 m+3}{(m+1)(2 m+1)}\left[\hat{N}_{2 m+1}(\beta l)+j \hat{J}_{2 m+1}(\beta l)\right]{\hat{J_{2 m+1}}}_{2 r}(\beta r)
$$


$I_{0}$ is the principal current amplitude on the transmission line, and $m$ is an integer. For $(2 n+1) \lambda / 2$ doublets in which we are interested (where $n=0,1,2,3, \ldots$ ), $I_{0} \cong I(0) . \quad \hat{J}_{2 \mathrm{~m}+1}(\beta l)$ and $\hat{N}_{2 \mathrm{~m}+1}(\beta l)$ are special functions related to $J$ and $N$ Bessel functions of order $n+1 / 2$ [1, p. 52].

The total relative current on the antenna is given by the sum of eq 34 and 35 . For an antenna composed of an infinitely thin filament $\left(K_{0}=\infty\right)$ the relative current distribution reduces to $\cos \beta r$, as is usually assumed for such a case.

\section{References}

[1] S. A. Schelkunoff, Electromagnetic waves, p. 290 to 292 and 441 to 479 (D. Van Nostrand Co., Inc., New York, N. Y., 1943).

[2] S. A. Schelkunoff, Theory of antennas of arbitrary size and shape, Proc. IRE 29, 508 (Sept. 1941).

[3] R. King, Graphical representation of the characteristics of cylindrical antennas, Cruft Laboratory, Tech. Rept. No. 20 (ONR) (Oct. 1, 1947).

[4] L. S. Nergaard, A survey of ultra-high-frequency measurements, RCA Rev. 3, 183 (Oct. 1938).

[5] MIT Radiation Laboratory Series-Crystal rectifiers, Louis N. Ridenour, editor, p. 333 to 360 (McGrawHill Book Co., New York, N. Y., 1948).

[6] Ernst A. Guillemin, Communication Networks II, 64 (John Wiley \& Sons Inc., New York, N. Y., 1935). [6a] R. E. Grantham, and J. J. Freeman, A Standard of Attenuation for Microwave Measurements, Trans. A. I. E. E., 67, p. 535, 1948.

[7] Nathand Marchand, Transmission-line conversion transformers, Electronics p. 142 to 145 (Dec. 1944).

[8] K. A. Norton, The propagation of radio waves over the earth and in the upper atmosphere, Proc. IRE 25, 1217 (Sept. 1937).

[9] K. A. Norton, The calculation of ground-wave field intensity over a finitely-conducting spherical earth, Proc. IRE 29, 623 to 639 (Dec. 1941).

[10] C. R. Burrows, Radio propagation over plane earthField strength curves, Bell System Tech. J. 16, 45 to 75 (Jan. 1937).

[11] K. Bullington, Radio propagation at frequencies above 30 megacycles, Proc. IRE 35, 1122 to 1136 (Oct. 1947).

[12] J. A. Stratton, Electromagnetie theory, p. 493 (McGraw-Hill Book Co., Inc., New York, N. Y., 1941).

[13] R. King, H. R. Mimno, A. H. Wing, Transmission lines, antennas, and waveguides, p. 160 (McGrawHill Book Co., Inc., New York, N. Y., 1945).

[14] Frank M. Greene, The influence of the ground on the calibration and use of VHF field-intensity meters, J. Research NBS 44, 123 (1950) RP2062.

Washington, October 3, 1949. 